\title{
Development and performance evaluation of an infinite horizon LQ optimal tracker
}

\author{
Péter Bauer ${ }^{\mathrm{a}, *}$, József Bokor ${ }^{\mathrm{a}}$ \\ ${ }^{a}$ Institute for Computer Science and Control, Hungarian Academy of Sciences, (MTA SZTAKI) \\ H-1111 Budapest, Kende u. 13-17. Hungary
}

\begin{abstract}
The paper presents an infinite horizon LQ optimal tracking control solution (LQ tracker) for discrete time linear time invariant systems. The reference preview need is reduced to only two steps irrespective of the type of reference signal making real-time implementation an achievable goal. A rigorous proof of optimality is provided for a set of infinite horizon reference commands which includes the linear combination of constant and exponentially bounded signals. Dissipativity, finite gain and $l_{1}$ performance of the controlled system are also evaluated. The behaviour of the proposed LQ tracker and its previously published sub-optimal version with one-step preview is demonstrated in conjunction with an application example. Their performances are compared to those of alternative solutions including set point control and model predictive control. Finally, it is concluded that the proposed rigorous solution of the infinite horizon tracking problem is real-time realizable and performs advantageously compared to other solutions.
\end{abstract}

Keywords:

Linear quadratic tracking control, Infinite horizon optimal tracker, Preview tracker, MPC tracker

\section{Introduction}

The research aiming to find real time realizable, finite or infinite horizon, linear quadratic (LQ) optimal tracking solutions started soon after the development

\footnotetext{
${ }^{*}$ Corresponding author, Tel.: +36-1-279-6163, Fax: +36-1-466-7483

Email addresses: bauer. peteresztaki.mta.hu (Péter Bauer), bokoresztaki.hu (József Bokor)
} 
This is the author version before final review of article published in European Journal of Control Vol. 39, January 2018, pp. 8-20

of LQ regulator theory, see e. g. Athans and Falb (1966). The finite horizon LQ optimal tracking problem is well established in the control literature, its treatment can be found in textbooks such as Athans and Falb (1966); Lewis (1986); Anderson and Moore (1989). However, all these solutions result in recursive Riccati and auxiliary differential equations. Thus they require the reference signal to be known for the entire future horizon which is a significant restriction in most of the practical applications. The infinite horizon (steady state) Riccati equation is well established, but the infinite horizon auxiliary equation can only be approximated so as to eliminate the need to know the reference over infinite time. To overcome this difficulty additional information about the reference can be considered making use of dynamical models such as in Anderson and Moore (1989); AlbaFlores and Barbieri (2006); Barbieri and Alba-Flores (2000) or a finite approximation and/or extrapolation of the reference signal can be applied as in Maciejowski (2002); Pachter and Miller (1998); Nagy (1999); Park et al. (2008).

In their earlier works (Bauer and Bokor, 2011; Bauer, 2013a,b) the authors of the present paper dealt with a strictly realizable solution for the discrete time infinite horizon LQ optimal tracking problem considering constant as well as timevarying references. In these works, only one-step preview was allowed, which led to a sub-optimal solution for constant and a set of time-varying references. The present work builds upon the results in literature and also upon the aforementioned results of the authors. It examines more deeply how to formulate a steady state auxiliary equation without the need for infinite horizon preview and how to satisfy the optimality criteria. Finally, it applies transformation of the controlled system with an assumed moving set point and finds a closed form solution with only twostep preview need. The idea of transformation comes from Willems and Mareels (2004) and Kwakernaak and Sivan (1972). The two-step preview need results directly from the structure of the centered auxiliary equation, the only restriction is that the moving set point is assumed to be known. It also proves the finiteness of the centered LQ cost function and the fulfilment of other optimality criteria for linear combination of constant and exponentially bounded reference signals. Dissipativity, finite gain and $l_{1}$ stability are examined also.

Finally, the proposed optimal infinite horizon LQ tracker and the cited suboptimal one Bauer $(2013 \mathrm{~b}, \mathrm{a})$ are compared to a nonzero set point tracker from Kwakernaak and Sivan (1972), a preview and a model predictive (MPC) controller from Farooq and Limebeer (2005) and Maciejowski (2002) respectively through an application example. These controllers with different theoretical basis are applied for the same system model with the same quadratic cost function and preview horizon to make the comparison 'fair'. For this reason the MPC control 
This is the author version before final review of article published in European Journal of Control Vol. 39, January 2018, pp. 8-20

horizon is also limited to two-steps.

\section{Problem statement}

Consider the following linear time invariant (LTI) discrete time (DT) system:

$$
\begin{aligned}
x_{k+1} & =A x_{k}+B \tilde{u}_{k} \\
y_{k} & =C x_{k}
\end{aligned}
$$

where $x \in \mathbb{R}^{n}, \tilde{u} \in \mathbb{R}^{m}$ and $y \in \mathbb{R}^{p}$ are the state, input and the output of the system respectively. It is assumed that their dimensions satisfy $p \leq m \leq n$ and the state matrices $A, B$ and $C$ have compatible dimensions. It is also assumed that the pair $(A, B)$ is stabilizable.

The goal of tracking control design is to follow a given reference signal ( $r \in$ $\mathbb{R}^{p}$ ) with the output $y$. Throughout the developments a pre-stabilization state feedback gain $K_{x 1}$ is selected from the set $\mathscr{K}$ by applying $\tilde{u}_{k}=-K_{x 1} x_{k}+u_{k}$ to (1) if required. If $A$ is Hurwitz then the pre-stabilization gain is not required.

$$
\mathscr{K}=\left\{K_{x 1}: \phi=A-B K_{x 1}, \phi \text { Hurwitz, } \operatorname{rank}\left(C(I-\phi)^{-1} B\right)=p\right\}
$$

The rank condition is required to later ensure the full row rank of the matrix $C(I-\phi)^{-1} B$. The finite horizon output tracking problem for (1) can be formulated using the following quadratic cost function published in Anderson and Moore (1989).

$$
\begin{aligned}
J_{N}(x, \tilde{x}, \tilde{u})= & \\
= & \frac{1}{2} \sum_{k=0}^{N-1}\left(\left(x_{k}-\tilde{x}_{k}\right)^{T} Q\left(x_{k}-\tilde{x}_{k}\right)+\tilde{u}_{k}^{T} R \tilde{u}_{k}\right)+ \\
& +\left(x_{N}-\tilde{x}_{N}\right)^{T} Q\left(x_{N}-\tilde{x}_{N}\right) \\
Q= & \bar{C}^{T} Q_{1} \bar{C}+C^{T} Q_{2} C \\
\tilde{x}_{k}= & C^{T}\left(C C^{T}\right)^{-1} r_{k}=H r_{k} \\
& Q_{2}>0, Q_{1} \geq 0, R>0
\end{aligned}
$$

Here, $J_{N}$ is the cost function for $N$ finite steps, $H=C^{T}\left(C C^{T}\right)^{-1}, \tilde{x}_{k}=H r_{k}$ is the reference state formulated from the reference signal and $\bar{C}=I-H C$ represents 
This is the author version before final review of article published in European Journal of Control Vol. 39, January 2018, pp. 8-20

the orthogonal projection of $x_{k}$ to the null space of $C$. The latter makes it possible to weight - through $Q_{1}$ - the states that are not affected by the tracking of $r_{k}$. This weighting can improve system performance.

The goal of finite horizon LQ optimal tracking control design is to choose $\tilde{u}_{k}, k=0 \ldots N-1$ to minimize the quadratic cost function $J_{N}$ subject to the dynamic constraint described in (1) (the $r$ reference and so $\tilde{x}$ in the cost can be considered as measured disturbances).

In the infinite horizon case the limiting solution $N \rightarrow \infty$ should be considered for the same problem.

\section{The finite horizon discrete time LQ optimal tracker}

After defining the tracking problem to be solved this section aims to summarize and improve the existing finite horizon solution. The finite horizon optimal solution can be obtained applying the Lagrange multiplier method for equations (3) and (1) and is well established in literature see e. g. Lewis (1986). The solution includes the well known discrete algebraic Ricatti equation with solution $P_{k}$ and the auxiliary equation with solution $v_{k}$. Both of them are obtained as recursive expressions with well defined final values:

$$
\begin{aligned}
P_{k} & =A^{T} P_{k+1}\left[I+B R^{-1} B^{T} P_{k+1}\right]^{-1} A+Q \\
v_{k} & =\left[A^{T}-A^{T} P_{k+1}\left[I+B R^{-1} B^{T} P_{k+1}\right]^{-1} B R^{-1} B^{T}\right] v_{k+1}+Q \tilde{x}_{k} \\
P_{N} & =Q, \quad v_{N}=Q \tilde{x}_{N}
\end{aligned}
$$

The resulting form of the costate variable (in this case the Lagrange multiplier) $\lambda_{k}$ and optimal control input is:

$$
\begin{aligned}
\lambda_{k}= & P_{k} x_{k}-v_{k} \\
\tilde{u}_{k}= & R^{-1} B^{T} \lambda_{k+1}= \\
= & -R^{-1} B^{T} P_{k+1}\left[I+B R^{-1} B^{T} P_{k+1}\right]^{-1} A x_{k}+ \\
& +R^{-1} B^{T}\left[I+P_{k+1} B R^{-1} B^{T}\right]^{-1} v_{k+1}
\end{aligned}
$$

However, the same derivation steps can lead to an extended costate variable structure which can be crucial in the infinite horizon solution and also satisfies equations in (4) and (5): 
This is the author version before final review of article published in European Journal of Control Vol. 39, January 2018, pp. 8-20

$$
\lambda_{k}=P_{k} x_{k}+S_{k} H r_{k+1}-Q H r_{k}=P_{k} x_{k}-v_{k}
$$

Matrix $H$ is defined in (3). The detailed derivation of the above expression is summarized in Appendix A. $S_{k}$ is the variable introduced into the extended auxiliary equation instead of $v_{k}$. The formulation presented in the appendix that results in the specific advantageuos structure - to the best knowledge of the authors - has not been presented in the literature yet.

\section{The infinite horizon, discrete time, LQ optimal tracker}

The goal of this section is to attempt to solve the infinite horizon tracking problem based on the improved finite horizon solution presented in the previous section. The infinite horizon solution (i. e., when $N \rightarrow \infty$ ) can be constructed based on Lewis (1986). It states that the optimal infinite horizon solution - the so called time-invariant tracker - can be obtained by substituting $P_{\infty}$ (i. e. the solution of the steady state discrete algebraic Riccati equation (DARE)) into all the expressions. The DARE is:

$$
P_{\infty}=A^{T} P_{\infty}\left[I+B R^{-1} B^{T} P_{\infty}\right]^{-1} A+Q
$$

Substituting $P_{\infty}$ into the costate variable and auxiliary equation one gets:

$$
\begin{gathered}
\lambda_{k}=P_{\infty} x_{k}+S_{k} H r_{k+1}-Q H r_{k}=P_{\infty} x_{k}-v_{k} \\
v_{k}=\left[A^{T}-A^{T} P_{\infty}\left[I+B R^{-1} B^{T} P_{\infty}\right]^{-1} B R^{-1} B^{T}\right] v_{k+1}+Q H r_{k}= \\
=A^{T} \underbrace{\left[I+P_{\infty} B R^{-1} B^{T}\right]^{-1}}_{M_{2}} v_{k+1}+Q H r_{k}
\end{gathered}
$$

Substituting $v_{k}=Q H r_{k}-S_{\infty} H r_{k+1}$ into the costate equation (9) (taking the limit $S_{\infty}=\lim _{k \rightarrow \infty} S_{k}$ only formally) one obtains:

$$
Q H r_{k}-S_{\infty} H r_{k+1}=Q H r_{k}+A^{T} M_{2}\left(Q H r_{k+1}-S_{\infty} H r_{k+2}\right)
$$


This is the author version before final review of article published in European Journal of Control Vol. 39, January 2018, pp. 8-20

From now, the only question that remains in conjunction with the solution is the existence of a steady state gain $S_{\infty}$ for the auxiliary equation (10). The equation is satisfied if the system of equations (11) is satisfied $\forall k \in \mathbb{N}$.

$$
\begin{aligned}
-S_{\infty} H r_{k+1} & =A^{T} M_{2} Q H r_{k+1} \\
0 & =-A^{T} M_{2} S_{\infty} H r_{k+2}
\end{aligned}
$$

If there exists an $S_{\infty}$ which satisfies the system of equations in (11) $\forall k$ then the control input constructed applying equations (5) and (8) turns out to be optimal and the preview need is reduced to only two-steps:

$$
\begin{aligned}
\tilde{u}_{k}= & -R^{-1} B^{T} P_{\infty}\left[I+B R^{-1} B^{T} P_{\infty}\right]^{-1} A x_{k}+ \\
& +R^{-1} B^{T}\left[I+P_{\infty} B R^{-1} B^{T}\right]^{-1} \underbrace{\left(Q H r_{k+1}-S_{\infty} H r_{k+2}\right)}_{v_{k+1}}
\end{aligned}
$$

At this point two problems arise. At first, equation (11) can not be satisfied for a nonzero $r_{k+2}$ as it requires $S_{\infty}=0$ or $r_{k+2}$ to be in the null space of $A^{T} M_{2} S_{\infty} H$. In case of $S_{\infty}=0$, the first equation in (11) can be satisfied only for $r_{k+1}=0$ alternatively $r_{k+1}$ must be in the null space of $A^{T} M_{2} Q H$. Both conditions are too restrictive. In case of $S_{\infty} \neq 0$ on the other hand it is impossible to achieve $A^{T} M_{2} S_{\infty} H r_{k+2}=0$ for arbitrary $r_{k+2}$.

Apart from satisfying equation (11) the other problem is related to the cost function. Optimality requires a finite cost function value in (3) on infinite horizon which can not be guaranteed even for a constant nonzero reference $r_{\infty}$. Tracking of nonzero set point usually requires a nonzero steady state control input and so $\tilde{u}_{k}^{T} R \tilde{u}_{k} \nrightarrow 0$ if $k \rightarrow \infty$. This drives the infinite horizon functional value $J_{\infty}(x, \tilde{x}, u)-$ obtained from $J_{N}$ by $N \rightarrow \infty$ - into infinity. The problem can possibly be solved by transformating the reference with an $r_{\infty_{k}}$ moving set point and correspondingly the system with the related $x_{\infty_{k}}$ state and $u_{\infty_{k}}$ input and defining the tracking problem for the transformed system. Considering the cost function, this means the removal of the energy related to the tracking of the moving set point. This moving set point dynamics can be described by a reference system (see the 2 nd point below) which is assumed to be in its steady state at every time step. The transformed system that is to be controlled LQ optimally, represents the difference between the set points and the real system (see 3rd point below) and so, the transient dynamics between them. If the moving set point covers the real reference signal, LQ optimal 
This is the author version before final review of article published in European Journal of Control Vol. 39, January 2018, pp. 8-20

regulation of the difference to zero means perfect tracking. As the difference state dynamics should approach zero the related output should also and this makes possible to get a finite cost functional value on infinite horizon (as it happens in the case of a simple LQ optimal regulator design).

As an illustration consider tracking of a constant nonzero reference signal $r_{\infty}$ which requires a nonzero $u_{\infty}$ input and this makes $J_{\infty}$ infinite. However, if the reference system tracks $r_{\infty}$ through $u_{\infty}$ the transformed system describes only the dynamics of the transient until the system reaches $r_{\infty}$. In this transient dynamics the states and the inputs should all approach zero and so, the infinite horizon tracking control is directly related to the infinite horizon regulator problem. The solution of this problem - called set point tracking - is well described in Kwakernaak and Sivan (1972) for example. This reformulation can possibly also help to achieve steady state of the auxiliary equation in (10).

In Bauer (2013a,b) a sub-optimal solution was derived based on a three-step procedure which comprises also a transformation step. The whole procedure is detailed below.

1. If the system matrix $A$ is not Hurwitz, choose a stabilizing state feedback gain from the set $\mathscr{K}$ and substitute $\tilde{u}_{k}=-K_{x 1} x_{k}+u_{k}$ into (1) to make step 2 solvable. This results in a stabilized system matrix $\phi$ as defined in (2):

$$
x_{k+1}=\underbrace{\left(A-B K_{x 1}\right)}_{\phi} x_{k}+B u_{k}, \quad y_{k}=C x_{k}
$$

If $A$ is Hurwitz, $\phi=A, K_{x 1}=0$ and $\tilde{u}_{k}=u_{k}$ can be applied.

2. Determine the state and input of the reference system related to $r_{\infty_{k}}$ assuming that this is a steady state set point for the stabilized system (13) followed by the system output at every time step as it is indicated in the second equation below.

$$
\begin{aligned}
& x_{\infty_{k}}=\phi x_{\infty_{k}}+B u_{\infty_{k}}, \rightarrow x_{\infty_{k}}=(I-\phi)^{-1} B u_{\infty_{k}} \\
& y_{\infty_{k}}=C x_{\infty_{k}}=r_{\infty_{k}} \\
& y_{\infty_{k}}=C(I-\phi)^{-1} B u_{\infty_{k}}=M u_{\infty_{k}}=r_{\infty_{k}}
\end{aligned}
$$

It should be noted that the inverse matrix $(I-\phi)^{-1}$ always exists because the $\phi$ Hurwitz matrix has all its eigenvalues inside the unit circle. The required 
This is the author version before final review of article published in European Journal of Control Vol. 39, January 2018, pp. 8-20

control input to hold $y_{\infty_{k}}=r_{\infty_{k}}$ is the solution of the last equation above which depends on the dimension of the matrix $M$. $M$ is a $p \times m$ matrix with $\operatorname{rank}(M)=\operatorname{rank}\left(C(I-\phi)^{-1} B\right)=p$ because $\phi$ is Hurwitz. If $p=m$ then equation (14) has a unique solution:

$$
u_{\infty_{k}}=\left(C(I-\phi)^{-1} B\right)^{-1} r_{\infty_{k}}=M^{-1} r_{\infty_{k}}
$$

If $p<m$ then equation (14) is underdetermined with infinitely many solutions. The input with minimum norm can be obtained applying the MoorePenrose pseudoinverse $\left(M^{+}\right)$as described e. g. in Demmel (1997). (In the rest of the article the inverse or if it does not exist the pseudoinverse of $M$ is denoted by $M^{+}$).

$$
u_{\infty_{k}}=\left(C(I-\phi)^{-1} B\right)^{+} r_{\infty_{k}}=M^{+} r_{\infty_{k}}
$$

3. Construct an LQ optimal tracking controller for the difference dynamics of the original system obtained around the moving set point $x_{\infty_{k}}, u_{\infty_{k}}, r_{\infty_{k}}$.

The difference dynamics, its infinite horizon cost function (with $\Delta \tilde{x}_{k}=H\left(r_{k}-\right.$ $\left.r_{\infty_{k}}\right)=H \Delta r_{k}$ ) and the related conditions of optimality can be formulated as follows by applying the $P_{\infty}$ steady state solution of the DARE.

$$
\begin{aligned}
x_{k+1}-x_{\infty_{k}} & =\phi\left(x_{k}-x_{\infty_{k}}\right)+B\left(u_{k}-u_{\infty_{k}}\right) \\
\Delta x_{k+1} & =\phi \Delta x_{k}+B \Delta u_{k}
\end{aligned}
$$

$$
\begin{aligned}
J_{\infty}(\Delta x, \Delta \tilde{x}, \Delta u) & = \\
& =\frac{1}{2} \sum_{k=0}^{\infty}\left(\left(\Delta x_{k}-\Delta \tilde{x}_{k}\right)^{T} Q\left(\Delta x_{k}-\Delta \tilde{x}_{k}\right)+\Delta u_{k}^{T} R \Delta u_{k}\right)
\end{aligned}
$$

$$
\begin{aligned}
\Delta \lambda_{k} & =P_{\infty} \Delta x_{k}+S_{k} H \Delta r_{k+1}-Q H \Delta r_{k}=P_{\infty} \Delta x_{k}-\Delta v_{k} \\
\Delta v_{k} & =Q H \Delta r_{k}-S_{k} H \Delta r_{k+1} \\
\Delta u_{k} & =-R^{-1} B^{T} \Delta \lambda_{k+1} \\
\Delta v_{k} & =\phi^{T} M_{2} \Delta v_{k+1}+Q H \Delta r_{k}
\end{aligned}
$$

The last equation above is the analogue of (9) and it is obtained by replacing $A^{T}$ with $\phi^{T}$ and applying the $\Delta$ notations. The critical tasks are to find a steady 
This is the author version before final review of article published in European Journal of Control Vol. 39, January 2018, pp. 8-20

state solution $\left(S_{\infty}\right)$ for the auxiliary equation (the last equation in (19) with $\Delta v_{k}$ and $\Delta v_{k+1}$ substituted) and prove the finiteness of the cost function in (18). Firstly, the possible steady state is examined, then the finiteness of the cost function is proven in the next section for a given set of references.

\subsection{Solution for constant references}

Assuming $r_{\infty_{k}}=r_{k}=$ const $\forall k$ results in $\Delta r_{k}=0, \Delta \tilde{x}_{k}=0 \quad \forall k$ and reduces the problem to regulation of the transformed system into the steady zero state. In this case $x_{\infty_{k}}=x_{\infty}, u_{\infty_{k}}=u_{\infty}$ and this represents a real steady state for the original system. This is a well established problem called set point control and its solution is proven to be optimal, see e. g. Kwakernaak and Sivan (1972).

\subsection{Solution for time-varying references}

For time-varying references the dynamics of the transformed auxiliary equation results from equations (19) and (10) as:

$$
Q H \Delta r_{k}-S_{\infty} H \Delta r_{k+1}=Q H \Delta r_{k}+\phi^{T} M_{2}\left(Q H \Delta r_{k+1}-S_{\infty} H \Delta r_{k+2}\right)
$$

This can be further generalized by defining $S_{1}=Q H$ and $S_{2}=S_{\infty} H$ as auxiliary unknown variables:

$$
S_{1} \Delta r_{k}-S_{2} \Delta r_{k+1}=Q H \Delta r_{k}+\phi^{T} M_{2}\left(S_{1} \Delta r_{k+1}-S_{2} \Delta r_{k+2}\right)
$$

The question is the selection of $r_{\infty_{k}}, S_{1}$ and $S_{2}$ to satisfy condition (21) $\forall k$. This requires the elimination of $\Delta r_{k+2}$ from the equation as it is explained related to equation (11) for the non-centered case. It is easy to see two options: the first is the approximation of $\Delta r_{k+2}$ based on other reference values, possibly $\Delta r_{k+1}$ and $\Delta r_{k}$ already included in (21). The second is to make $\Delta r_{k+2}$ zero through the selection of $r_{\infty_{k}}$.

The first approach was applied in Bauer $(2013 a, b)$ with the linear extrapolation of $\Delta r_{k+2}=2 \Delta r_{k+1}-\Delta r_{k}$. There, the assumption $r_{\infty_{k}}=r_{k+1}$ was used. This way a closed form sub-optimal solution was obtained with the following gains:

$$
\begin{aligned}
& S_{1}=\left[I-\phi^{T} M_{2}\left(\left(I-\phi^{T} M_{2}\right)^{2}\right)^{-1} \phi^{T} M_{2}\right] Q H \\
& S_{2}=-\left(\left(I-\phi^{T} M_{2}\right)^{2}\right)^{-1} \phi^{T} M_{2} Q H
\end{aligned}
$$


This is the author version before final review of article published in European Journal of Control Vol. 39, January 2018, pp. 8-20

This is only a sub-optimal solution as at the next time step the extrapolation $\Delta r_{k+3}=2 \Delta r_{k+2}-\Delta r_{k+1}$ includes $\Delta r_{k+2}$. So, $\Delta r_{k+2}$ is assumed known at that time step, but was extrapolated before. This means that two different values are considered for the same reference in two consecutive time steps and so there are step by step differences from the real reference signal. For this reason the solution can not be optimal for the original reference signal.

In the present work the second approach is used with $r_{\infty_{k}}=r_{k+2}$ and so $\Delta r_{k+2}=$ $r_{k+2}-r_{\infty_{k}}=0$ without any approximation (extrapolation) of the reference signal. The resulting gains are then:

$$
S_{1}=Q H, \quad S_{2}=-\phi^{T} M_{2} Q H
$$

This is valid $\forall k$ but requires two-step preview of future reference. It should be emphasized that, compared to the infinite horizon preview need this can lead to real-time realizability.

Reformulating (12) for the centered system dynamics and considering the generalized $\Delta v_{k+1}$ with gains $S_{1}$ and $S_{2}$ the control input for the centered system is as follows $\left(\Delta r_{k+2}=0\right)$ :

$$
\begin{aligned}
\Delta u_{k}= & -\underbrace{R^{-1} B^{T} P_{\infty}\left[I+B R^{-1} B^{T} P_{\infty}\right]^{-1} \phi}_{K_{x 2}} \Delta x_{k}+ \\
& +\underbrace{R^{-1} B^{T}\left[I+P_{\infty} B R^{-1} B^{T}\right]^{-1} S_{1}}_{K_{r}} \Delta r_{k+1}
\end{aligned}
$$

Here, $K_{x 2}$ is the well-known infinite horizon LQ optimal state feedback gain. Expanding $\Delta x_{k}$ and $\Delta r_{k+1}$, substituting the expressions of $u_{\infty_{k}}$ and $x_{\infty_{k}}$ from equation (14) and considering the pre-stabilization of the system $\left(K_{x 1}\right)$ (in case if $A$ was Hurwitz $K_{x 1}=0$ can be considered, but $K_{x 1}$ will be included in all forthcoming formulae for notational conveniance) and $r_{\infty_{k}}=r_{k+2}$, one gets the final input $\tilde{u}_{k}$ as:

$$
\begin{aligned}
& u_{k}=-K_{x 2} x_{k}-K_{r}\left(r_{k+2}-r_{k+1}\right)+\left(K_{x 2}(I-\phi)^{-1} B+I\right) M^{+} r_{k+2} \\
& \tilde{u}_{k}=-\underbrace{\left(K_{x 1}+K_{x 2}\right)}_{K_{x}} x_{k}-K_{r}\left(r_{k+2}-r_{k+1}\right)+\underbrace{\left(K_{x 2}(I-\phi)^{-1} B+I\right) M^{+}}_{K_{r_{\infty}}} r_{k+2}
\end{aligned}
$$

The above equations represent a $\mathrm{PD}$-like $(\mathrm{PD}=$ proportional and derivative) control solution with respect to the reference signal. As the reference signal is 
This is the author version before final review of article published in European Journal of Control Vol. 39, January 2018, pp. 8-20

usually noiseless this control formulation is more advantegous than the conventional PD control which utilizes the tracking error. The above control provides fast reaction for reference changes meanwhile removes the problem with noise amplification. The anti-windup problem is also eliminated because there is no integral action in the controller.

\section{Proof of infinite horizon optimality}

After obtaining an infinite horizon solution for the LQ optimal tracking problem its most important properties are summarized in a theorem and proven tehereafter.

Theorem 1. The control input $\tilde{u}_{k}$ in (25) which results from the three-step solution proposed in Section 4 gives an infinite horizon LQ optimal output tracker with only a two-step reference preview need for a set of time-varying references (which can be bounded by the sum of exponentially convergent and constant signals) for the system described in (1) with cost function given in (18).

PROOF. The constant reference case i. e. set point tracking is well established in literature, see e. g. Kwakernaak and Sivan (1972). For this reason the optimality should be proven only for the case of time-varying references. The conditions of optimality - resulting from Lagrange multiplier method - are given in equations (19) which should be completed with the proof of the finiteness of (18).

Unfortunately it is not possible to prove optimality for any arbitrary infinite horizon time-varying signal because frequent increments (decrements) in the reference signal will frequently change the transformed system and so does not let its dynamics to converge to zero (reach the moving set point). Summing up small but infinitely many tracking error terms will drive the functional value infinite and so will violate a condition of optimality.

However, considering practical applications, only finite time bounded reference signals should be included in the proof of optimality for infinite horizon time-varying references because every system works only on a finite time horizon. Such a finite time bounded signal can be always upper bounded by the sum of a constant $r_{c}$ and a time-varying exponentially convergent $r_{k}$ signal (leading to $r=r_{c}+r_{k}$ ) as Figure 1 shows. This exponential bound can be considered as a worst case description of the reference signal. Proving the optimality for this class of signals is possible as shown in the sequel 
This is the author version before final review of article published in European Journal of Control Vol. 39, January 2018, pp. 8-20

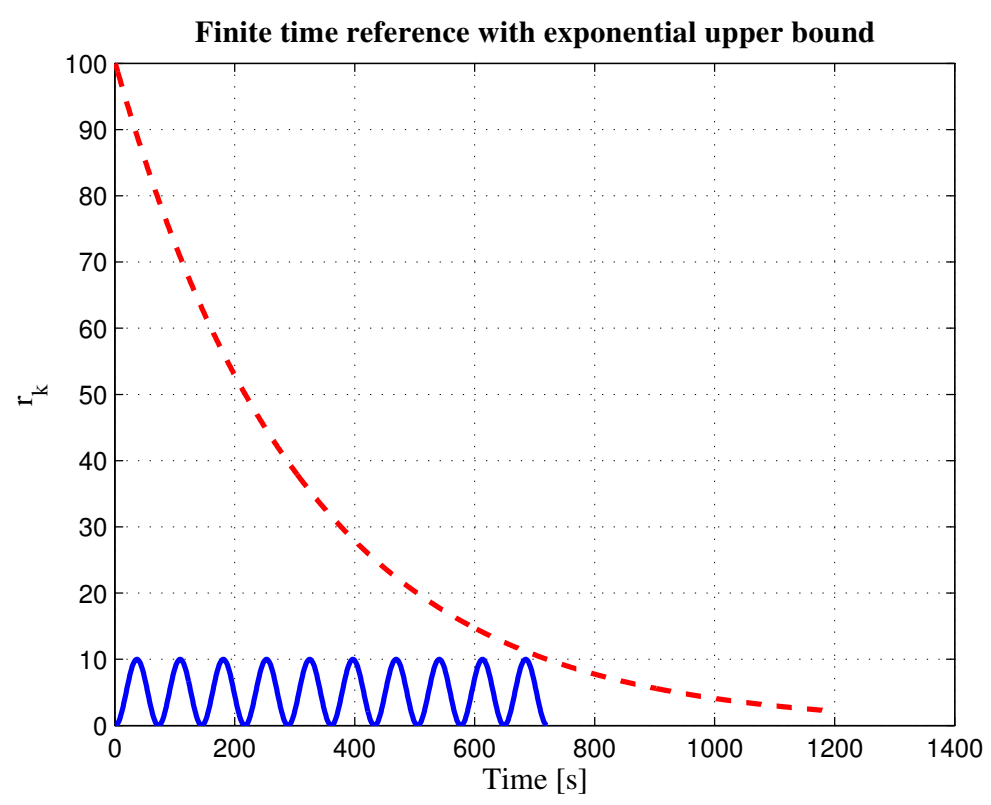

Figure 1: Finite time bounded reference signal with its worst case upper bound

The tracking of the two reference parts can be examined separately due to the linearity of the system. The optimality of the constant reference tracking part was mentioned above. In this subsection only the optimality with exponentially bounded references will be proven. The exponential bound of the reference can be formulated as below:

$$
\left|r_{k}\right|<E e^{-a k}, E \in \mathbb{R}^{+}, a \in \mathbb{R}^{+} \backslash\{0\}, \lim _{k \rightarrow \infty} r_{k}=0
$$

Considering the conditions of optimality given in equations (19) the centered control input was formulated in equation (24) by substituting the proposed structure of the costate with $S_{1}, S_{2}$ and $r_{\infty_{k}}=r_{k+2}$. So the optimal input equation in (19) is satisfied.

The $P_{\infty}$ solution of DARE again satisfies the algebraic Riccati equation in the limiting case.

The only aspects to be proven are if the auxiliary equation is satisfied and the infinite horizon cost function has a finite value.

The $S_{1}$ and $S_{2}$ values were derived from the transformed auxiliary equation shown in (21) by considering reference values $r_{k}, r_{k+1}, r_{k+2}$ and $r_{\infty_{k}}=r_{k+2}$ (at 
This is the author version before final review of article published in European Journal of Control Vol. 39, January 2018, pp. 8-20

time $k$ ) without any constraint on $k$. So, the obtained solution is valid for every $k=[0, \infty)$.

The last aspect is the finiteness of the centered infinite horizon cost function $J_{\infty}$ in (18) with moving set point values:

$$
\begin{aligned}
r_{\infty_{k}} & =r_{k+2} \\
u_{\infty_{k}} & =M^{+} r_{k+2} \\
x_{\infty_{k}} & =(I-\phi)^{-1} B M^{+} r_{k+2}=M_{\infty} r_{k+2}
\end{aligned}
$$

In Bauer $(2013 \mathrm{a}, \mathrm{b})$ finiteness of the centered $J_{\infty}$ cost function shown in (18) with $r_{\infty_{k}}=0$ and exponentially bounded references is proven. This derivation is extended here to nonzero $r_{\infty_{k}}$ values. Note that the extension published here could also be done for the solution of Bauer $(2013 \mathrm{a}, \mathrm{b})$ which uses extrapolation of $r_{k+2}$ and $r_{\infty_{k}}=r_{k+1}$. However, this extension of previous results is out of the scope of this article.

Consider the stabilized system dynamics as described in (13) together with its derived control input $u_{k}$ from (25). Substituting $u_{k}$ into (13) results in:

$$
x_{k+1}=\underbrace{\left(\phi-B K_{x 2}\right)}_{\phi_{1}} x_{k}-B K_{r} \underbrace{\left(r_{k+2}-r_{k+1}\right)}_{\Delta \bar{r}_{k+2}}+B K_{r_{\infty}} r_{k+2}
$$

The state dynamics and transformation with moving set point results in the expressions given below. First, $x_{k+2}$ is formulated according to (28), then $x_{k+1}$ is substituted into it also from (28). $\Delta x_{k+2}$ is obtained by subtracting $x_{\infty_{(k+2)}}$ from $x_{k+2}$ considering equation (27). Finally, generalization of the $x_{k+2}$ formula for $x_{k}$ with shifted indices and $x_{0}$ initial value and the subtraction of $x_{\infty_{(k)}}$ gives the last equation in (29). 
This is the author version before final review of article published in European Journal of Control Vol. 39, January 2018, pp. 8-20

$$
\begin{aligned}
x_{k+2}= & \phi_{1} x_{k+1}-B K_{r} \underbrace{}_{\Delta \bar{r}_{k+3}\left(r_{k+3}-r_{k+2}\right)}+B K_{r_{\infty}} r_{k+3}= \\
= & \phi_{1}^{2} x_{k}-\phi_{1} B K_{r} \Delta \bar{r}_{k+2}-B K_{r} \Delta \bar{r}_{k+3}+\phi_{1} B K_{r_{\infty}} r_{k+2}+B K_{r_{\infty}} r_{k+3} \\
\Delta x_{k+2}= & \phi_{1}^{2} x_{k}-\phi_{1} B K_{r} \Delta \bar{r}_{k+2}-B K_{r} \Delta \bar{r}_{k+3}+ \\
& +\phi_{1} B K_{r_{\infty}} r_{k+2}+B K_{r_{\infty}} r_{k+3}-\underbrace{M_{\infty} r_{k+4}}_{x_{\infty}(k+2)} \\
\Delta x_{k}= & \phi_{1}^{k} x_{0}-\underbrace{\sum_{l=0}^{k-1} \phi_{1}^{l} B K_{r} \Delta \bar{r}_{k+1-l}}_{\Delta R_{k}}+\underbrace{\sum_{l=0}^{k-1} \phi_{1}^{l} B K_{r_{\infty}} r_{k+1-l}}_{L_{k}}-M_{\infty} r_{k+2}
\end{aligned}
$$

Considering equation (24) $\Delta u_{k}$ can be expressed with $\Delta x_{k}$ :

$$
\Delta u_{k}=-K_{x 2} \phi_{1}^{k} x_{0}+K_{x 2} \Delta R_{k}-K_{x 2} L_{k}+K_{x 2} M_{\infty} r_{k+2}+K_{r} \Delta r_{k+1}
$$

The only term left to deal with from the cost function is $\Delta \tilde{x}_{k}=H \Delta r_{k}=H\left(r_{k}-\right.$ $\left.r_{\infty_{k}}\right)=H\left(r_{k}-r_{k+2}\right)$. Substituting all the $\Delta$ expressions into cost function $J_{\infty}$ in equation (18), furthermore defining $F=Q+K_{x 2}^{T} R K_{x 2}$ and expanding all the multiplications results in the following expression: 
This is the author version before final review of article published in European Journal of Control Vol. 39, January 2018, pp. 8-20

$$
\begin{aligned}
J_{\infty}(\Delta x, \Delta \tilde{x}, \Delta u)= & 1 \\
= & 2 \sum_{k=0}^{\infty}(\underbrace{x_{0}^{T}\left(\phi_{1}^{T}\right)^{k} F \phi_{1}^{k} x_{0}}_{\text {Term } 1}+\underbrace{\Delta R_{k}^{T} F \Delta R_{k}}_{\text {Term } 2}+\underbrace{L_{k}^{T} F L_{k}}_{\text {Term } 3}+ \\
& +\underbrace{\underbrace{T}_{k+2} M_{\infty}^{T} F M_{\infty} r_{k+2}}_{\text {Term } 4}-\underbrace{2 x_{0}^{T}\left(\phi_{1}^{T}\right)^{k} F \Delta R_{k}}_{\text {Term } 5}+\underbrace{2 x_{0}^{T}\left(\phi_{1}^{T}\right)^{k} F L_{k}}_{\text {Term } 6}- \\
& -\underbrace{2 x_{0}^{T}\left(\phi_{1}^{T}\right)^{k} F M_{\infty} r_{k+2}}_{\text {Term } 7}-\underbrace{2 \Delta R_{k}^{T} F L_{k}}_{\text {Term } 8}+\underbrace{2 \Delta R_{k}^{T} F M_{\infty} r_{k+2}}_{\text {Term } 9}- \\
& -\underbrace{2 L_{k}^{T} F M_{\infty} r_{k+2}}_{\text {Term } 10}-\underbrace{2 x_{0}^{T}\left(\phi_{1}^{T}\right)^{k} Q H \Delta r_{k}}_{\text {Term } 11}+\underbrace{2 \Delta R_{k}^{T} Q H \Delta r_{k}}_{\text {Term } 12}- \\
& -\underbrace{2 L_{k}^{T} Q H \Delta r_{k}}_{\text {Term } 13}+\underbrace{2 r_{k+2}^{T} M_{\infty}^{T} Q H \Delta r_{k}}_{\text {Term } 14}+\underbrace{\Delta r_{k}^{T} H^{T} Q H \Delta r_{k}}_{\text {Term } 15}+ \\
& +\underbrace{2 x_{0}^{T}\left(\phi_{1}^{T}\right)^{k} K_{x 2}^{T} R K_{r} \Delta \bar{r}_{k+2}}_{\text {Term } 16}-\underbrace{2 \Delta R_{k}^{T} K_{x 2}^{T} R K_{r} \Delta \bar{r}_{k+2}}_{\text {Term } 17}+ \\
& +\underbrace{\Delta \bar{r}_{k+2}^{T} K_{r}^{T} R K_{r} \Delta \bar{r}_{k+2}}_{\text {Term } 20})
\end{aligned}
$$

This cost function is an infinite series. Such a series has a finite limit if the absolute series constructed from it has a finite limit. This statement is proven in Appendix B. Based on the proof given there one can state that the derived infinite horizon LQ tracking solution is indeed optimal for the sum of exponentially bounded and constant references.

In the next section some stability metrics are considered to proof stability and finiteness of the tracking error with the proposed LQ optimal tracker.

\section{Dissipativity, finite gain and $l_{1}$ stability of the tracking error}

Having proven the optimality of the proposed tracking method, the convergence (stability) of its tracking error should be examined. The resulting system after feedback with state matrix $\phi_{1}$ (see equation (28)) is guaranteed to be stable, however the stability of the tracking error for any type of input should be checked. 
This is the author version before final review of article published in European Journal of Control Vol. 39, January 2018, pp. 8-20

As a first step derive the dynamics of the tracking error. Considering (14) and (16) the tracking error at time $k+1$ is:

$$
e_{k+1}=y_{k+1}-r_{k+1}=C x_{k+1}-C \underbrace{(I-\phi)^{-1} B M^{+}}_{M_{\infty}} r_{k+1}=C \underbrace{\left(x_{k+1}-M_{\infty} r_{k+1}\right)}_{\hat{x}_{k+1}}
$$

The next step is to formulate the dynamics of the $\hat{x}$ error state. From equations (28), (25) and from the above equation the error state dynamics can be expressed as:

$$
\begin{aligned}
& \hat{x}_{k+1}=x_{k+1}-M_{\infty} r_{k+1}= \\
& =\underbrace{\left(\phi-B K_{x 2}\right)}_{\phi_{1}} x_{k}-B K_{r} \underbrace{\left(r_{k+2}-r_{k+1}\right)}_{\Delta \bar{r}_{k+2}}+B K_{r_{\infty}} r_{k+2}-M_{\infty} r_{k+1} \\
& B K_{r_{\infty}}=B K_{x 2} M_{\infty}+B M^{+}=B K_{x 2} M_{\infty}+(I-\phi)(I-\phi)^{-1} B M^{+}= \\
& =-\phi_{1} M_{\infty}+M_{\infty} \\
& \hat{x}_{k+1}=\phi_{1}(x_{k}-M_{\infty} \quad \underbrace{r_{k+2}})-\left(B K_{r}-M_{\infty}\right)\left(r_{k+2}-r_{k+1}\right)= \\
& r_{k}+\Delta \bar{r}_{k+1}+\Delta \bar{r}_{k+2} \\
& =\phi_{1} \hat{x}_{k}+\underbrace{\left[\begin{array}{ll}
-\phi_{1} M_{\infty} & \left(I-\phi_{1}\right) M_{\infty}-B K_{r}
\end{array}\right]}_{\bar{B}} \underbrace{\left[\begin{array}{c}
\Delta \bar{r}_{k+1} \\
\Delta \bar{r}_{k+2}
\end{array}\right]}_{\overline{\Delta r}_{k}}
\end{aligned}
$$

The error state dynamics together with the tracking error formulates a strictly causal dynamical system:

$$
\begin{aligned}
\hat{x}_{k+1} & =\phi_{1} \hat{x}_{k}+\bar{B} \overline{\Delta r}_{k} \\
e_{k} & =C \hat{x}_{k}
\end{aligned}
$$

Unfortunately it is not possible to obtain a Lyapunov function for the tracking error. This is because the system is not autonomous and the unknown input - the reference signal - is continuously changing in time. However, from Kottenstette et al. (2014) considering $V_{k}=\hat{x}_{k}^{T} P \hat{x}_{k}$ as the storage function and

$$
s\left(e_{k}, \overline{\Delta r}_{k}\right)=\left[\begin{array}{ll}
e_{k}^{T} & \overline{\Delta r}_{k}
\end{array}\right]\left[\begin{array}{cc}
Q & S \\
S^{T} & R
\end{array}\right]\left[\frac{e_{k}}{\Delta r_{k}}\right]
$$


This is the author version before final review of article published in European Journal of Control Vol. 39, January 2018, pp. 8-20

as the quadratic supply rate (where $Q=Q^{T}$ and $R=R^{T}$ ) the generalization of the positive real lemma provides the opportunity to check the dissipativity and other stability properties.

$$
\left[\begin{array}{cc}
\phi_{1}^{T} P \phi_{1}-P-C^{T} Q C & \phi_{1}^{T} P \bar{B}-C^{T} S \\
\bar{B}^{T} P \phi_{1}-S^{T} C & \bar{B}^{T} P \bar{B}-R
\end{array}\right] \leq 0
$$

The LQ optimal tracking solution is dissipative if the above linear matrix inequality (LMI) is satisfied for any (Q,S,R). If it is satisfied for $Q=-I, S=0, R=$ $\gamma^{2} I$ then the LQ tracker is also finite gain stable. Passivity can be proven if the LMI is satisfied for $Q=0, S=\frac{1}{2} I, R=0$. This can be checked only for quadratic systems. In our case $\operatorname{dim}(e)=p$ and $\operatorname{dim}(\overline{\Delta r})=2 p \neq p$ that's why passivity can not be examined.

Another possibility is to examine the $l_{1}$ gain of the error system representing the gain for the $l_{\infty}$ norm of $\overline{\Delta r}_{k}$ which is the maximum step in the reference signal between two samples. This $l_{1}$ gain can be calculated based on the Markov parameters of the system as Jochen M. Rieber (2007) describes:

$$
\begin{aligned}
\mathscr{G}(k) & =C \phi_{1}^{k-1} \bar{B}, \quad k \geq 1 \\
\|\mathscr{G}\|_{1} & =\max _{1 \leq i \leq p} \sum_{j=1}^{2 p} \sum_{k=1}^{\infty}\left|\mathscr{G}_{i j}(k)\right|
\end{aligned}
$$

For $k=0$ the Markov parameter is $D=0$ and so can be excluded. Dimensions $p$ and $2 p$ of $\mathscr{G}$ correspond to the dimension of $e$ and $\overline{\Delta r}$ respectively. In numerical evaluation $\|\mathscr{G}(k)\|_{F}$ (i. e. the Frobenius norm of $\mathscr{G}(k)$ matrix) decreases below machine precision upon $k>N>0$ since $\phi_{1}$ is a stable matrix. Therefore $\|\mathscr{G}\|_{1}$ can be evaluated in finite number of steps.

Numerical evaluation of all of the above stability metrics appear near the end of Section 8. In the next section three other tracking solutions are formulated so that the proposed LQ tracker can be compared to these. The sub-optimal LQ tracker solution derived in Bauer $(2013 \mathrm{a}, \mathrm{b})$ is also compared to all the other solutions.

\section{Other tracking methods for comparison}

Having derived the above LQ tracking solution (from now on we will refer to this as $L Q T$ ), it should be compared to other existing solutions on a fair basis. This 
This is the author version before final review of article published in European Journal of Control Vol. 39, January 2018, pp. 8-20

means that the other solutions should be considered for the same centered system given in (17) with $r_{\infty_{k}}=r_{k+2}$ moving set point and for the same cost function as given in (18) and considering maximum two- step preview horizon.

\subsection{Nonzero set point tracking}

For nonzero set point tracking (SPT) the solution of Kwakernaak and Sivan (1972) is applied:

$$
\tilde{u}_{k}=-K_{x} x_{k}+K_{r_{\infty}} r_{k+2}
$$

This SPT can be considered as a P-like control with only one term for the reference signal and with exactly the same $K_{x}$ and $K_{r_{\infty}}$ gains as for the LQ tracker.

\subsection{Preview tracking control}

This preview tracking $(P V)$ solution can be easily derived considering $N=2$ preview horizon based on Farooq and Limebeer (2005) as follows:

$$
\begin{aligned}
& \Delta x_{k}^{r}=\left[\begin{array}{lll}
\Delta r_{k}^{T} & \Delta r_{k+1}^{T} & \Delta r_{k+2}^{T}
\end{array}\right]^{T} \\
& \Delta x_{k+1}^{r}=\underbrace{\left[\begin{array}{lll}
0 & I & 0 \\
0 & 0 & I \\
0 & 0 & 0
\end{array}\right]}_{A_{d}} \Delta x_{k}^{r}+\underbrace{\left[\begin{array}{l}
0 \\
0 \\
I
\end{array}\right]}_{B_{d}} \Delta r_{k+3} \\
& \Delta r_{k}=\underbrace{\left[\begin{array}{lll}
I & 0 & 0
\end{array}\right]}_{C_{d}} \Delta x_{k}^{r} \\
& \Delta x_{k}^{a}=\left[\begin{array}{ll}
\Delta x_{k}^{T} & \left(\Delta x_{k}^{r}\right)^{T}
\end{array}\right]^{T} \\
& \Delta x_{k}-\Delta \tilde{x}_{k}=\left[\begin{array}{ll}
I & -H C_{d}
\end{array}\right] \Delta x_{k}^{a}=C_{a} \Delta x_{k}^{a} \\
& J_{P V}\left(\Delta x^{a}, \Delta u\right)=\frac{1}{2} \sum_{k=0}^{\infty}\left(\left(\Delta x_{k}^{a}\right)^{T} C_{a}^{T} Q C_{a} \Delta x_{k}^{a}+\Delta u_{k}^{T} R \Delta u_{k}\right) \\
& \Delta u_{k}=-K_{x 2} \Delta x_{k}-K_{r} \Delta x_{r}(k)= \\
& =-K_{x 2} \Delta x_{k}-K_{r 1} \Delta r_{k}-K_{r 2} \Delta r_{k+1}-K_{r 3} \Delta r_{k+2} \\
& r_{\infty_{k}}=r_{k+2} \rightarrow \\
& \tilde{u}_{k}=-K_{x} x_{k}+K_{r 1}\left(r_{k+2}-r_{k}\right)+K_{r 2}\left(r_{k+2}-r_{k+1}\right)+K_{r_{\infty}} r_{k+2}
\end{aligned}
$$

This is a PD-like solution with two difference terms and with exactly the same $K_{x}$ and $K_{r_{\infty}}$ gains as for the LQ tracker. 
This is the author version before final review of article published in European Journal of Control Vol. 39, January 2018, pp. 8-20

\subsection{Model predictive control}

The unconstrained, closed form model predictive control $(M P C)$ solution was derived again with $N=2$ horizon according to Maciejowski (2002). The horizon was limited to two-steps to make the solution comparable to the aforementioned LQT method.

$$
\begin{aligned}
& J_{M P C}(\Delta x, \Delta \tilde{x}, \Delta u, k)= \\
& =\frac{1}{2}\left(\sum_{i=1}^{2}\left(\Delta x_{k+i}-\Delta \tilde{x}_{k+i}\right)^{T} Q\left(\Delta x_{k+i}-\Delta \tilde{x}_{k+i}\right)+\right. \\
& \left.+\sum_{j=0}^{1} \Delta u_{k+j}^{T} R \Delta u_{k+j}\right) \\
& J_{M P C}(k)=\frac{1}{2}\left(\left\|Z_{k}-T_{k}\right\| \frac{2}{Q}+\left\|U_{k}\right\|_{\frac{2}{R}}\right) \\
& Z_{k}=\left[\begin{array}{l}
\Delta x_{k+1} \\
\Delta x_{k+2}
\end{array}\right] \quad T_{k}=\left[\begin{array}{l}
\Delta \tilde{x}_{k+1} \\
\Delta \tilde{x}_{k+2}
\end{array}\right] \quad U_{k}=\left[\begin{array}{c}
\Delta u_{k} \\
\Delta u_{k+1}
\end{array}\right] \\
& Z_{k}=\left[\begin{array}{c}
\phi \\
\phi^{2}
\end{array}\right] \Delta x_{k}+\left[\begin{array}{cc}
B & 0 \\
\phi B & B
\end{array}\right] U_{k}=\gamma \Delta x_{k}+\Theta U_{k} \\
& \bar{Q}=\left[\begin{array}{ll}
Q & 0 \\
0 & Q
\end{array}\right] \quad \bar{R}=\left[\begin{array}{ll}
R & 0 \\
0 & R
\end{array}\right] \\
& M_{M}=\left(\Theta^{T} \bar{Q} \Theta+\bar{R}\right)^{-1} \Theta^{T} \bar{Q} \\
& U_{k}=M_{M}\left(T_{k}-\gamma \Delta x_{k}\right) \quad M_{M}=\left[\begin{array}{ll}
M_{11} & M_{12} \\
M_{21} & M_{22}
\end{array}\right] \\
& \Delta u_{k}=M_{11} H \Delta r_{k+1}+M_{12} H \Delta r_{k+2}-\underbrace{\left(M_{11} \phi+M_{12} \phi^{2}\right)}_{K_{x 2}^{(M P C)}} \Delta x_{k} \\
& r_{\infty_{k}}=r_{k+2} \rightarrow \\
& \tilde{u}_{k}=-K_{x}^{(M P C)} x_{k}-M_{11} H\left(r_{k+2}-r_{k+1}\right)+K_{r_{\infty}}^{(M P C)} r_{k+2}
\end{aligned}
$$

Here, $\|x\|_{Q}^{2}$ symbolizes the quadratic form $x^{T} Q x$. The resulting control input represents a PD-like solution with only one difference term and with $K_{x}^{(M P C)}=$ $K_{x 1}+K_{x 2}^{(M P C)}$ and $K_{r_{\infty}}^{(M P C)}=\left(K_{x 2}^{(M P C)}(I-\phi)^{-1} B+I\right) M^{+}$gains which are different from those of LQT, SPT and PV. 
This is the author version before final review of article published in European Journal of Control Vol. 39, January 2018, pp. 8-20

\subsection{Results with extrapolated $r_{k+2}$}

To be able to compare the proposed methods to the sub-optimal tracker derived in Bauer (2013a,b), their inputs are also derived with extrapolation of the reference signal $\left(\Delta r_{k+2}=2 \Delta r_{k+1}-\Delta r_{k}\right)$ and different set point $\left(r_{\infty_{k}}=r_{k+1}\right)$ (call these solutions as EX (extrapolated) and the others in the previous subsections as NEX (non-extrapolated)). The summary of EX control inputs for the different methods can be seen below.

1. Non-zero set point (SPT): $\tilde{u}_{k}=-K_{x} x_{k}+K_{r_{\infty}} r_{k+1}$

2. Preview control (PV): $\tilde{u}_{k}=-K_{x} x_{k}+\left(K_{r 1}-K_{r 3}\right)\left(r_{k+1}-r_{k}\right)+K_{r_{\infty}} r_{k+1}$

3. MPC control (MPC): $\tilde{u}_{k}=-K_{x}^{(M P C)} x_{k}+M_{12} H\left(r_{k+1}-r_{k}\right)+K_{r_{\infty}}^{(M P C)} r_{k+1}$

These are structurally similar solutions, but with different reference signal values. The two difference terms in the preview solution are unified with the $\left(K_{r 1}-K_{r 3}\right)$ gain.

\section{Application example}

After deriving the infinite horizon LQ tracking solution and presenting three other well known tracking solutions in the previous sections an application example is presented. The goal with the example is to numerically compare the performance of the solutions. The second example from the work Park et al. (2008) was chosen to be implemented because it fits the need to present tracking results on a higher dimensional (four states, two inputs) but not overly complicated system. The example is further related to the topic as it was applied to test receding horizon LQ tracking control in Park et al. (2008). The parameters of the longitudinal dynamical model of the Navion aircraft presented in Park et al. (2008) were converted to SI units with $v_{f}$ forward, $v_{d}$ downward velocities $[\mathrm{m} / \mathrm{s}], \theta$ pitch angle $[\mathrm{rad}], \delta_{e}$ elevator deflection $[\mathrm{rad}]$ and $\delta_{T}$ specific thrust $\left[\mathrm{m} / \mathrm{s}^{2}\right]$. The state space model of the system with converted parameters is presented in (42). 
This is the author version before final review of article published in European Journal of Control Vol. 39, January 2018, pp. 8-20

$$
\begin{aligned}
x & =\left[\begin{array}{llll}
v_{f} & v_{d} & \dot{\theta} & \theta
\end{array}\right]^{T} u=\left[\begin{array}{lll}
\delta_{e} & \delta_{T}
\end{array}\right]^{T} y=\theta \\
A & =\left[\begin{array}{cccc}
-0.045 & 0.036 & 0 & -9.81 \\
-0.37 & -2.02 & 53.645 & 0 \\
6.26 e-3 & -0.13 & -2.98 & 0 \\
0 & 0 & 1 & 0
\end{array}\right] \\
B & =\left[\begin{array}{cccc}
0 & -8.6 & -11 & 0 \\
1 & 0 & 0 & 0
\end{array}\right]^{T} C=\left[\begin{array}{llll}
0 & 0 & 0 & 1
\end{array}\right]
\end{aligned}
$$

The discrete time equivalent of this system was obtained and used in the control with $100 \mathrm{~Hz}$ sampling frequency. The goal was to track pitch angle references with the controllers (LQT, SPT, PV and MPC). Both the EX and NEX methods were tested. The references are a doublet series, and a chirp signal $(0-0.5 \mathrm{~Hz})$, both with $\pm 10^{\circ}$ (or $\pm 0.1745 \mathrm{rad}$ ) amplitude. The tracking results and so the references are presented in Figures 2 and 4.

The control inputs are limited by saturation to comply with the capabilities of the Navion aircraft. The maximum deflections of the elevator are $-30^{\circ}$ up $1+20^{\circ}$ down so the symmetrical limit is $\pm 0.349 \mathrm{rad}$. The maximum specific thrust is $1.543 \mathrm{~m} / \mathrm{s}^{2}$ while in the considered trim point (cruise with $176 \mathrm{ft} / \mathrm{s}$ ) it is $0.863 \mathrm{~m} / \mathrm{s}^{2}$. This means that its maximum change can be $\pm 0.68 \mathrm{~m} / \mathrm{s}^{2}$ (all of this data is approximately calculated).

The control weights were selected considering the cost function $J_{\infty}$ defined in (18). $Q_{1}$ weights the states that has not been affected by the tracking of the output signal. In these particular case these states are the velocities $v_{f}, v_{d}$ and the pitch rate $\dot{\theta} \cdot Q_{1}=0$ was selected in all cases to provide the best possible tracking of $\theta$ references (not to limit the system dynamics with further constraints). The initial $R$ input weight was selected based on Bryson's rule (method of inverse squares):

$$
R=<1 / 0.349^{2}, 1 / 0.68^{2}>=<8.21,2.16>
$$

Here, $\langle\ldots\rangle$ symbolizes that $R$ is a diagonal matrix with the given values in its main diagonal. Later $R$ was decreased to obtain better tracking results. The final input weight is $R=<0.0080 .002>$ in all cases.

The $Q_{2}$ tracking weight was tuned in every case (quantized to the multiples of ten) to obtain the best tracking results. In most of the cases different weights were required to attain minimum cost function value or minimum average tracking error. These different cases are denoted herein by $\min C$ for minimum cost function 
This is the author version before final review of article published in European Journal of Control Vol. 39, January 2018, pp. 8-20

and $\min T$ for minimum average tracking error, respectively. The final weight values after tuning are summarized in Appendix C.

For the purpose of comparison amongst the different methods, the following performance measures - for the finite simulation horizon - were used:

1. Average absolute tracking error: $\theta_{e}=\left(\sum_{k=0}^{N}\left|\theta_{k}-r_{k}\right|\right) \frac{1}{N}$

2. Value of the cost (18) with saturated control inputs, finite time horizon and the following moving setpoint settings (referenced as $J_{S}$ in the sequel):

$$
\begin{array}{r}
\text { for NEX method } r_{\infty_{k}}=r_{k+2} \rightarrow u_{\infty_{k}}=M^{+} r_{k+2} \text { and } x_{\infty_{k}}=M_{\infty} r_{k+2} \\
\text { for EX method } r_{\infty_{k}}=r_{k+1} \rightarrow u_{\infty_{k}}=M^{+} r_{k+1} \text { and } x_{\infty_{k}}=M_{\infty} r_{k+1}
\end{array}
$$

3. Value of the cost including tracking error and control energy relative to the moving $u_{\infty_{k}}$ value presented below:

$$
\begin{aligned}
J_{s 1}(y, u) & = \\
& =\frac{1}{2} \sum_{k=0}^{N}\left(\left(y_{k}-r_{k}\right)^{T}\left(y_{k}-r_{k}\right)+\Delta u_{k}^{T} \Delta u_{k}\right) \\
\text { for NEX method } r_{\infty_{k}} & =r_{k+2} \rightarrow u_{\infty_{k}}=M^{+} r_{k+2} \\
\text { for EX method } r_{\infty_{k}} & =r_{k+1} \rightarrow u_{\infty_{k}}=M^{+} r_{k+1}
\end{aligned}
$$

4. Value of the cost function including tracking error, system (the states excluding $\theta$ ) and control energy:

$$
\begin{aligned}
J_{s 2}(y, x, u) & = \\
& =\frac{1}{2} \sum_{k=0}^{N}\left(\left(y_{k}-r_{k}\right)^{T}\left(y_{k}-r_{k}\right)+x_{k}^{T} \bar{C}^{T} \bar{C} x_{k}+\Delta u_{k}^{T} \Delta u_{k}\right) \\
r_{\infty_{k}} & =r_{k+2} \rightarrow u_{\infty_{k}}=M^{+} r_{k+2} \text { for } N E X \\
r_{\infty_{k}} & =r_{k+1} \rightarrow u_{\infty_{k}}=M^{+} r_{k+1} \text { for } E X
\end{aligned}
$$

5. Average time delay $(\tau)$ in the tracking of the chirp signal obtained from the correlation function between the system output and the reference signal.

The numerical values of the above performance measures for the considered different cases are summarized in Appendix D.

The tracking results with the proposed NEX method are plotted in Figures 2 to 5. The results with EX method look very similar and therefore they are not included herein. 
This is the author version before final review of article published in European Journal of Control Vol. 39, January 2018, pp. 8-20

In the tracking of doublet signal (see Figure 3) the MPC method has a large overshoot compared to the others. The results obtained by the other three methods are exactly the same. This can be seen also in the tabular performance data (see Tables D.1 and D.2) where the LQT, SPT and PV errors and cost function values are the same both for NEX and EX methods. Note that all three methods were weighted similarly for doublet tracking as shown in table C.1. This similar behaviour is not surprising if one considers that the applied doublet reference is a piecewise constant signal and for constant signals the LQT, SPT and PV methods give exactly the same system input (assuming the same weighting). This is because the $K_{x}$ and $K_{r_{\infty}}$ gains are the same, and all the difference terms are zero for constant references. Therefore, only some minor differences could occur due to the step changes in the doublet reference signal. For this reason only the LQT and MPC solutions are plotted.

The average tracking errors with MPC were considerably larger than that of the other methods as a result of the large overshoots.

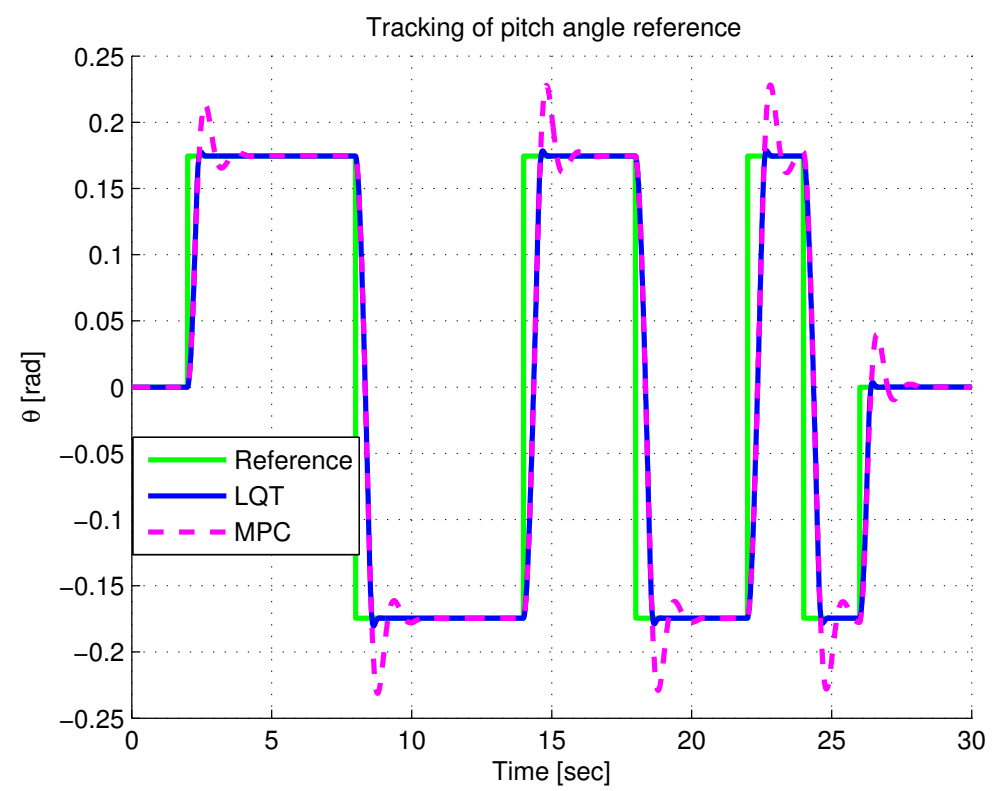

Figure 2: Tracking of pitch angle $(\theta)$ doublet series 
This is the author version before final review of article published in European Journal of Control Vol. 39, January 2018, pp. 8-20

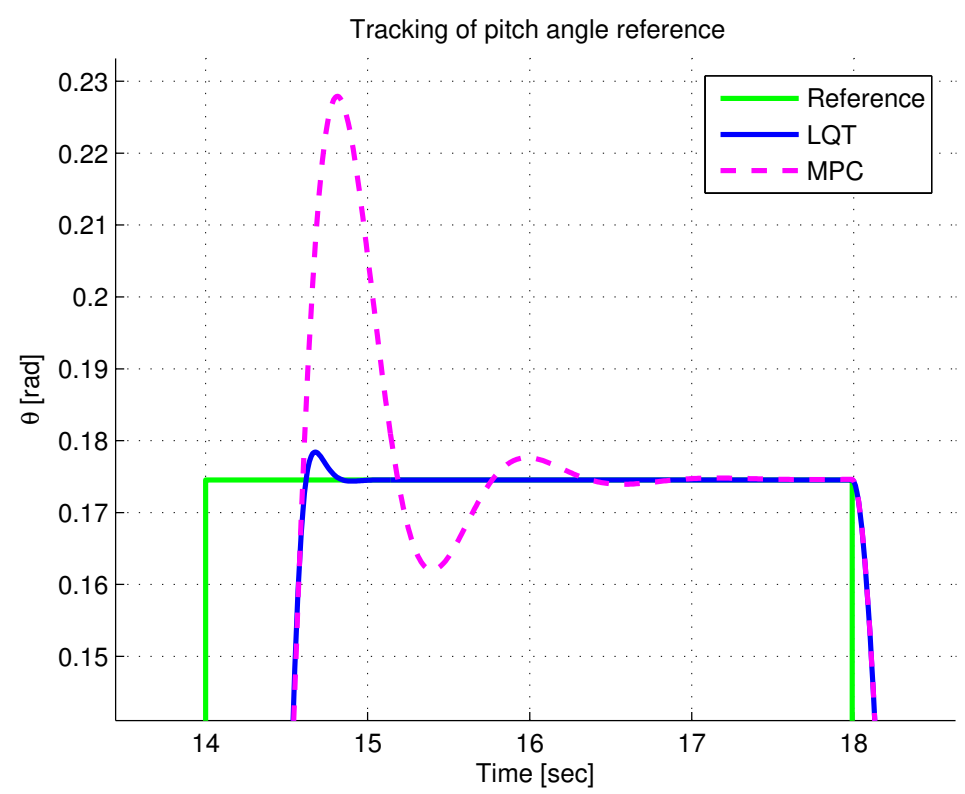

Figure 3: Tracking of pitch angle $(\theta)$ doublet series (enlarged from Figure 2)

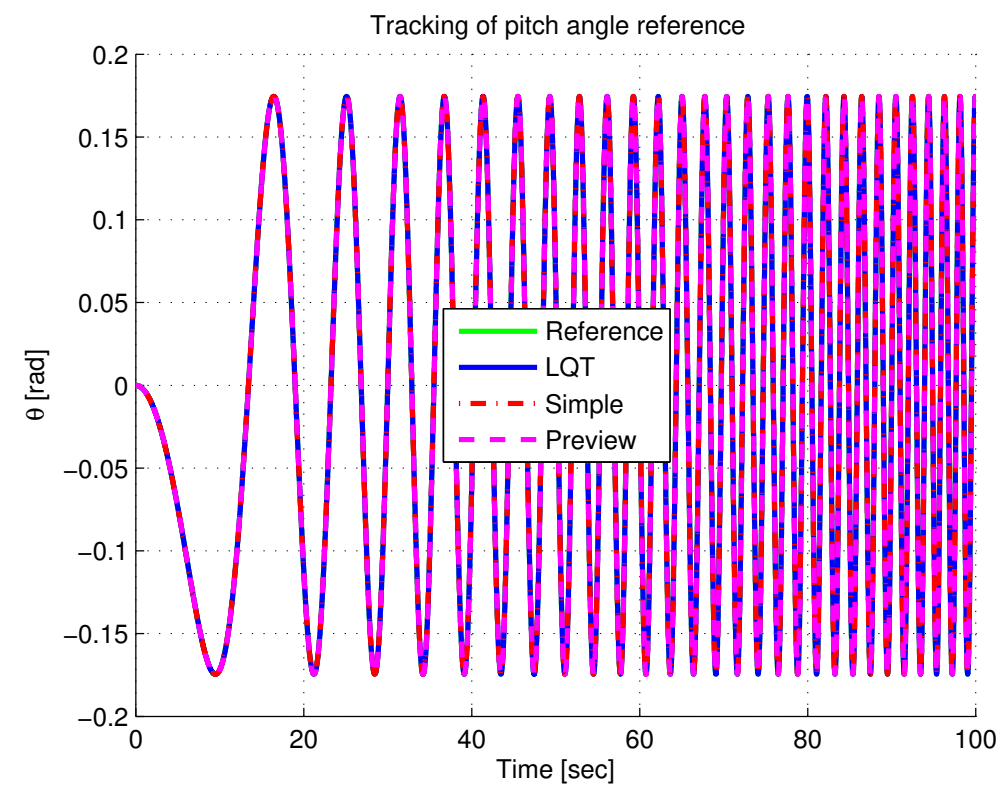

Figure 4: Tracking of pitch angle $(\theta)$ chirp signal $(0-0.5 \mathrm{~Hz})$ 
This is the author version before final review of article published in European Journal of Control Vol. 39, January 2018, pp. 8-20

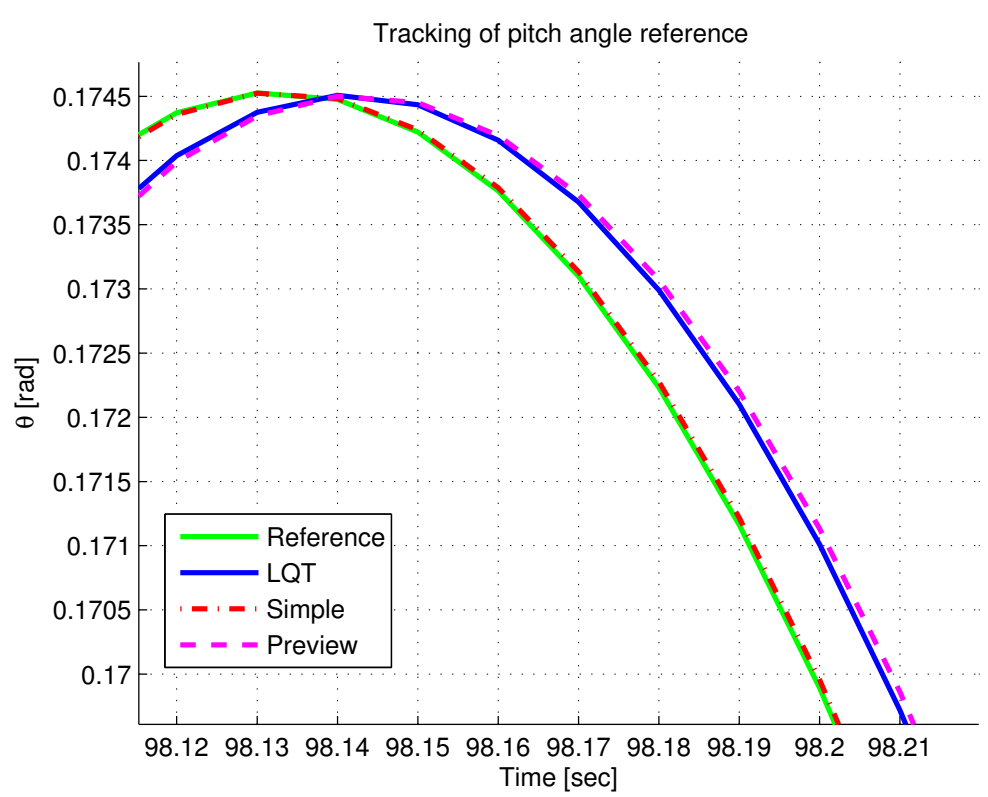

Figure 5: Tracking of pitch angle $(\theta)$ chirp signal $(0-0.5 \mathrm{~Hz})$ (enlarged from Figure 4)

The average tracking errors and $J_{s i}(i=1,2)$ cost values were smaller with NEX methods than with EX also for the MPC solution, so one can conclude that the knowledge of $r_{k+2}$ at time $k$ leads to better results. There is a negligible difference between the $J_{s 1}$ and $J_{s 2}$ costs in the NEX and EX cases. This can be explained again by the piecewise constant reference signal which removes significance of preview or extrapolation.

In chirp signal tracking, all the methods are tuned separately for minimum $J_{S}$ cost and minimum average tracking error. The minimization of time delay between reference and system output was also carried out. This limits the achievable lowest cost and means that there is a design trade-off between minimum cost and time delay.

Considering now Figure 5 the LQT and PV results are very close to each other, furthermore the MPC results are not plotted, because they completely match the LQT result. For chirp signal tracking the SPT NEX controller gives the best result. Tuning of the SPT method showed that by increasing the $Q_{2}$ weight the average tracking error can be decreased to an arbitrarily small value. Clearly, the related $J_{s}$ cost function value is very large but $J_{s 1}$ and $J_{s 2}$ are only negligibly larger than their associated values with the other methods.

The NEX methods give smaller average errors and cost in most of the cases, 
This is the author version before final review of article published in European Journal of Control Vol. 39, January 2018, pp. 8-20

but the results with EX are also acceptable.

\subsection{Evaluation of the stability metrics}

Stability metrics were calculated for the proposed LQT NEX method. The LMI's for the dissipativity and the finite gain stability were all feasible for the cases tuned for doublet tracking or chirp signal tracking, so the proposed solution is both dissipative and finite gain stable.

Considering the $l_{1}$ norm $\|\mathscr{G}\|_{1}=7.739$ resulted considering the tuning for doublet tracking and $\|\mathscr{G}\|_{1}=0.3698$ resulted for the tining for chirp signal tracking. The latter is an excellent $l_{1}$ norm which means that the system produces lower tracking errors than the greatest step in the reference signal. The former shows possibly much larger error than in the greatest change of the reference signal. However, in case of the doublet signal there is nonzero $\Delta r$ reference change only in few time steps otherwise $\overline{\Delta r}_{k}=0$ and therefore the problem simplifies to setpoint tracking which is proven in the literature to be stable (see e. g. Kwakernaak and Sivan (1972)). For this reason its tuning with finally $Q_{2}=10$ does not result in less than $1 l_{1}$ norm. On the contrary the tuning of the chirp signal tracking finally applied $Q_{2}=238370$ weight which is much larger.

Considering the $\|\cdot\|_{\infty}$ norms of the reference signal and the tracking error in case of the doublet input $\left\|\overline{\Delta r}_{k}\right\|_{\infty}=20^{\circ}$ and $\left\|e_{k}\right\|_{\infty}=19.989^{\circ}$ which well satisfies $\left\|e_{k}\right\|_{\infty}<\|\mathscr{G}\|_{1}\left\|\overline{\Delta r}_{k}\right\|_{\infty}$. On the contrary for the chirp signal $\left\|\overline{\Delta r}_{k}\right\|_{\infty}=0.3473^{\circ}$ and $\left\|e_{k}\right\|_{\infty}=0.3092^{\circ}$. This does not satisfy $\left\|e_{k}\right\|_{\infty}<\|\mathscr{G}\|_{1}\left\|\overline{\Delta r}_{k}\right\|_{\infty}$. The cause can be the larger time lag on higher frequencies, because the maximum tracking error continuously increases as the frequency of the reference signal increases.

\subsection{Overall evaluation of methods}

The performance of the compared methods varies with the considered application cases. MPC is the worst in most of the cases which is reasonable because of the finite (and very short) horizon cost function considered in its design.

The other three methods behave similarly for piecewise constant references. For chirp references when $r_{k+2}$ is assumed to be known (i. e. the NEX case) the SPT method is the best and the LQT and the PV perform similarly. Notably they have even the same optimal weighting when tuned for minimum tracking error (see table C.1). The $J_{s 1}$ and $J_{s 2}$ cost function values are very similar in all cases. In case of extrapolation of $r_{k+2}$ (EX case) the PV method gives the smallest average error while the SPT gives the largest. Even in these cases the cost values are very similar. 
This is the author version before final review of article published in European Journal of Control Vol. 39, January 2018, pp. 8-20

As a summary, it can be stated that the LQT, SPT and PV methods perform very similarly when they are tuned for best tracking performance. Neither of them can be identified as the best solution.

The main consequence is that the sub-optimal (EX) solution with only $r_{k+1}$ known (and $r_{k+2}$ extrapolated) can perform close to the optimal method with known $r_{k+2}$. This means that also the sub-optimal solution is quite suitable in practical applications if the optimal solution is not viable e. g. $r_{k+2}$ is unknown at time $k$.

\section{Conclusion}

This article deals with discrete time infinite horizon LQ optimal tracking control for LTI systems. It first references the finite horizon optimal solution from literature then improves it with the derivation of the detailed structure of the costate variable.

In the next major step it discusses the problems related to the infinite horizon solution such as the existence of steady state auxiliary equation without infinite horizon reference preview need and the finiteness of the cost function.

Two possible solutions are proposed to obtain a steady state auxiliary equation. They decrease the reference preview need to only one or two-steps. The solution with one-step preview is only sub-optimal and was published earlier in (Bauer $(2013 \mathrm{a}, \mathrm{b}))$ on the other hand the solution proposed here with two-step preview is optimal and is a new development.

If two-step reference preview is not possible then the one-step solution can provide a feasible alternative as the evaluation of the application example suggests. The knowledge of $r_{k+1}$ at time $k$ is mandatory for DT control feasibility so, the one-step solution is always viable.

The finiteness of the quadratic cost function is proven for a set of references including sum of exponentially bounded and constant signals. This set includes all the finite time bounded signals. In Bauer (2013a,b) a similar proof is presented by assuming $r_{\infty_{k}}=0$ moving set point. The present work extends the results published there by considering a nonzero moving set point $r_{\infty_{k}}=r_{k+2}$. This extension is applicable also for the $r_{\infty_{k}}=r_{k+1}$ case considered in Bauer (2013a,b).

Besides proving optimality, the stability metrics such as dissipativity, finite gain and $l_{1}$ gain stability are derived and examined.

Three other tracking solutions are derived for the same transformed system with same LQ cost function and preview horizon to render the comparison pos- 
This is the author version before final review of article published in European Journal of Control Vol. 39, January 2018, pp. 8-20

sible. For this purpose the set point control, the preview and MPC control are considered.

The developed infinite horizon optimal solution and the previously published sub-optimal one (Bauer (2013a,b)) are compared to the other three methods through an application example. The task in the example is to track piecewise constant and chirp references.

All of the methods were tuned to achieve their best tracking performance. The optimal ones with two-step preview give better results than the sub-optimal ones with one-step preview. But the quantitative difference is fairly small and so, the sub-optimal solutions with one-step preview are also well applicable if two-step preview is not viable.

The considered four control solutions presented in the paper, namely the proposed LQ tracker, the set point control, the preview and the MPC can not be ranked considering their performance only. To summarize the differences in the approaches, the set point control assumes a constant reference and solves a regulation problem for the centered system, the preview control considers a given preview horizon, extends the state space accordingly and solves the regulation problem, while MPC uses a finite horizon cost function. So only the proposed LQ tracker(s) solve the infinite horizon tracking problem in a rigorous way considering also the additional auxiliary equation. The presented solution is proven to be optimal and also practically viable. Its sub-optimal version with reduced preview need provides similar performance and therefore it can be advantageously applied if two-step reference preview is - for some reason - not possible.

The proposed tracking control formulation is sensitive to disturbances and uncertainties. As a solution to this problem an infinite horizon minimax tracker including unknown input estimation is derived in papers Bauer et al. (2009a), Bauer et al. (2009b) and Bauer et al. (2009b). The solutions proposed in these articles remove the adverse effect of disturbances. Sensitivity to uncertainties is examined in depth in Bauer (2013a) and Bauer (2013b) through stochastic performance and robustness analysis.

Future work may include examining the possible extensions of the method for linear parameter varying and nonlinear systems. In these cases, the difficulty lies in the handling of the moving setpoint and moving system parameters.

\section{Acknowledgement}

The authors gratefully acknowledge the reviewers work with several valuable comments which helped to improve the quality of the paper. The contribution of 
This is the author version before final review of article published in European Journal of Control Vol. 39, January 2018, pp. 8-20

professor Peter J. Seiler, Raghu Venkataraman and Bin Hu through preliminary discussions of this article is also acknowledged here. Their comments and questions has led to significant improvements of the work. The careful proof reading and several valuable suggestions of Zoltán Fazekas are also greatly acknowledged. This work was carried out at MTA (Hungarian Academy of Sciences) Institute for Computer Science and Control.

\section{References}

Alba-Flores, R., Barbieri, E., October 8-11 2006. Real-time Infinite Horizon Linear-Quadratic Tracking Controller for Vibration Quenching in Flexible Beams. In: Proc. of IEEE Conference on Systems, Man, and Cybernetics. Taipei, Taiwan, pp. 38-43.

Anderson, B., Moore, J., 1989. Optimal Control, Linear Quadratic Methods. Prentice Hall, Englewood Cliffs, NJ.

Athans, M., Falb, P., 1966. Optimal Control, An Introduction to the Theory and its Applications. McGraw-Hill Book Company.

Barbieri, E., Alba-Flores, R., 2000. On the Infinite-horizon LQ Tracker. Systems and Control Letters 40, 77-82.

Bauer, P., 2013a. Optimal Tracking Control for Unmanned Aerial Vehicles. Ph.D. thesis, Budapest University of Technology and Economics.

URL http://www.omikk.bme.hu/collections/phd/ Kozlekedesmernoki_es_Jarmumernoki_Kar/2013/

Bauer_Peter/ertekezes.pdf (18. December 2014.)

Bauer, P., 2013b. Optimal tracking solutions applied to unmanned aerial vehicles. LAP LAMBERT Academic Publishing.

Bauer, P., Bokor, J., November 2011. Infinite horizon LQ optimal output tracking from development to real flight tests. In: In Proc. of 12th IEEE International Symposium on Computational Intelligence and Informatics (CINTI 2011). Budapest, Hungary, pp. 277-282.

Bauer, P., Kulcsár, B., Bokor, J., 2009a. Discrete time minimax tracking control with disturbance estimation. In: Proc. of European Control Conference (ECC) 2009. Budapest, Hungary, pp. 2658-2663. 
This is the author version before final review of article published in European Journal of Control Vol. 39, January 2018, pp. 8-20

Bauer, P., Kulcsár, B., Bokor, J., 2009b. Discrete time minimax tracking control with state and disturbance estimation II: time-varying reference and disturbance signals. In: Proc. of IEEE 17th Mediterranean Conference on Control and Automation. Thessaloniki, Greece, pp. 486-491.

Demmel, J. W., 1997. Applied Numerical Linear Algebra. Society of Industrial and Applied Mathematics (SIAM).

Farooq, A., Limebeer, D., 2005. Path Following of Optimal Trajectories Using Preview Control. In: Proc. of 44th IEEE Conference on Decision and Control, and the European Control Conference 2005. Seville, Spain, pp. 2787-2792.

Jochen M. Rieber, 2007. Control of Uncertain Systems with $\uparrow_{1}$ and Quadratic Performance Objectives. Ph.D. thesis, University of Stuttgart.

Kottenstette, N., McCourt, M. J., Xia, M., Gupta, V., Antsaklis, P. J., 2014. Relationships Among Passivity, Positive Realness, and Dissipativity with an Application to Passivity Based Pairing. Tech. Rep. ISIS-2014-003, University of Notre Dame.

Kwakernaak, H., Sivan, R., 1972. Linear Optimal Control Systems. WileyInterscience.

Lewis, F. L., 1986. Optimal Control. John Wiley \& Sons.

Maciejowski, J. M., 2002. Predictive Control with Constraints. Pearson Education Limited.

Nagy, E., 1999. State Tracking through Optimized Trajectory Tracking. In: Proc. of American Control Conference. San Diego, California, USA, pp. 1807-1811.

Pachter, M., Miller, R. B., 1998. Manual Flight Control with Saturating Actuators. IEEE Control Systems Magazine 18 (1), 10-19.

Park, J. H., Han, S., Kwon, W. H., 2008. LQ tracking controls with fixed terminal states and their application to receding horizon controls. Systems and Control Letters 57, 772-777.

Willems, J. L., Mareels, I., 2004. A Rigorous Solution of the Infinite Time Interval LQ Problem with Constant State Tracking. Systems and Control Letters 52, 289-296. 
This is the author version before final review of article published in European Journal of Control Vol. 39, January 2018, pp. 8-20

\section{Appendix A. Derivation of the extended costate variable}

This section includes the detailed derivation of the extended costate variable and serves as a support material for section 3. Consider the nonhomogeneous Hamiltonian system consistent with the derivatives of the Lagrangian obtained from equations (1) and (3) based on Lewis (1986).

$$
\left[\begin{array}{c}
x_{k+1} \\
\lambda_{k}
\end{array}\right]=\left[\begin{array}{cc}
A & -B R^{-1} B^{T} \\
Q & A^{T}
\end{array}\right]\left[\begin{array}{c}
x_{k} \\
\lambda_{k+1}
\end{array}\right]+\left[\begin{array}{c}
0 \\
-Q H
\end{array}\right] r_{k}
$$

The equations from (A.1) for $k=N-1$ result in (considering the final condition $\left.\lambda_{N}=Q x_{N}-Q \tilde{x}_{N}\right)$ :

$$
\begin{aligned}
x_{N}= & A x_{N-1}-B R^{-1} B^{T} Q x_{N}+B R^{-1} B^{T} Q H r_{N} \\
\lambda_{N-1}= & Q x_{N-1}+A^{T} Q x_{N}-A^{T} Q H r_{N}-Q H r_{N-1} \\
x_{N}= & {\left[I+B R^{-1} B^{T} Q\right]^{-1}\left(A x_{N-1}+B R^{-1} B^{T} Q H r_{N}\right) } \\
\lambda_{N-1}= & Q x_{N-1}+A^{T} Q\left[I+B R^{-1} B^{T} Q\right]^{-1} A x_{N-1}+ \\
& +A^{T} Q\left[I+B R^{-1} B^{T} Q\right]^{-1} B R^{-1} B^{T} Q H r_{N}-A^{T} Q H r_{N}-Q H r_{N-1}
\end{aligned}
$$

From the last equation in (A.2) by introducing generalized and unknown gains $P$ and $S$ for the terms $x$ and $H r$, respectively, the extended structure of the costate variable results in:

$$
\begin{aligned}
\lambda_{N-1} & =P_{N-1} x_{N-1}+S_{N-1} H r_{N}-Q H r_{N-1} \\
\lambda_{k} & =P_{k} x_{k}+S_{k} H r_{k+1}-Q H r_{k}=P_{k} x_{k}-v_{k}
\end{aligned}
$$

It is worth noting that if one considers $v_{k}=-S_{k} H r_{k+1}+Q H r_{k}$ the equation above is completely consistent with the shorter form published in literature.

\section{Appendix B. Proof of the finiteness of the infinite horizon cost function for a set of time-varying references}

This section includes the details of the proof of optimality presented in Section 5. Finiteness of the infinite horizon cost function for exponentially bounded references is proven here via the finiteness of the sum of absolute series obtained 
This is the author version before final review of article published in European Journal of Control Vol. 39, January 2018, pp. 8-20

from (31). The absolute value of the sum of terms is upper bounded by the sum of absolute terms for every time step $k$. Therefore, the finite limit of the component series constructed from the absolute terms must be proven. If all these limits are finite, then their sum and so the limit of the cost function $J_{\infty}$ is also finite.

\section{Appendix B.1. Preliminary results}

- Firstly, the upper bound of the sum $\sum_{k=0}^{\infty}\left\|\phi_{1}^{k}\right\|$ is derived. In the following, $\|$. $\|$ denotes the induced $l_{2}$ norm (i. e. the maximum gain) of a matrix.

If the system matrix $\phi_{1}$ is stable with eigenvalues inside the unit circle, then there exists an index $K$ for which $\mu=\left\|\phi_{1}^{K}\right\|<1$. From this statement the limit shown below can be derived. For details see Bauer (2013a,b). The statement implies the existence of an upper bound $\phi_{U} \geq\left\|\phi_{1}^{k}\right\| \forall k$ for the sequence $\left\|\phi_{1}^{k}\right\|$.

$$
\sum_{k=0}^{\infty}\left\|\phi_{1}^{k}\right\|<\underbrace{\sum_{k=0}^{2 K-1}\left\|\phi_{1}^{k}\right\|}_{<\infty}+K \sum_{l=1}^{\infty} \mu^{l}=\sum_{k=0}^{2 K-1}\left\|\phi_{1}^{k}\right\|+\frac{K}{1-\mu}=K_{\phi}<\infty
$$

- Second, the upper bounds for reference signal related terms can be obtained considering equation (26):

$$
\begin{aligned}
& \left|\Delta \bar{r}_{k}\right|=\left|r_{k}-r_{k-1}\right| \leq\left|r_{k}\right|+\left|r_{k-1}\right|=E e^{-a k}+E e^{-a(k-1)}<2 E e^{-a(k-1)} \\
& \left|\Delta r_{k}\right|=\left|r_{k}-r_{k+2}\right| \leq\left|r_{k}\right|+\left|r_{k+2}\right|=E e^{-a k}+E e^{-a(k+2)}<2 E e^{-a k}
\end{aligned}
$$

- The upper bounds for $\left|\Delta R_{k}\right|$ and $\left|L_{k}\right|$ are obtained as below: 
This is the author version before final review of article published in European Journal of Control Vol. 39, January 2018, pp. 8-20

$$
\begin{aligned}
\left|\Delta R_{k}\right| & =\left|\sum_{l=0}^{k-1}\left(\phi_{1}^{l} B K_{r} \Delta \bar{r}_{k+1-l}\right)\right| \leq \sum_{l=0}^{k-1}\left\|\phi_{1}^{l}\right\|\left\|B K_{r}\right\|\left|\Delta \bar{r}_{k+1-l}\right|< \\
& <\sum_{l=0}^{k-1}\left\|\phi_{1}^{l}\right\|\left\|B K_{r}\right\| 2 E e^{-a(k-l)}<\phi_{U}\left\|B K_{r}\right\| 2 E \sum_{l=0}^{k-1} e^{-a(k-l)}< \\
& <\phi_{U}\left\|B K_{r}\right\| 2 E \frac{1}{1-e^{-a}}
\end{aligned}
$$

Derived analogously for $\left|L_{k}\right|$ :

$$
\left|L_{k}\right|<\phi_{U}\left\|B K_{r_{\infty}}\right\| E \frac{1}{1-e^{-a}}
$$

The limits can be derived term by term in a similar fashion. Here, for similar terms only the results are given.

Term 1:

$$
\begin{aligned}
& \frac{1}{2} \sum_{k=0}^{\infty}\left|x_{0}^{T}\left(\phi_{1}^{T}\right)^{k} F \phi_{1}^{k} x_{0}\right| \leq \frac{1}{2} \sum_{k=0}^{\infty}\left|x_{0}^{T}\right|\left\|\left(\phi_{1}^{T}\right)^{k}\right\|\|F\|\left\|\phi_{1}^{k}\right\|\left|x_{0}\right|= \\
& =\left|x_{0}\right|^{2}\|F\| \frac{1}{2} \sum_{k=0}^{\infty}\left(\left\|\left(\phi_{1}^{T}\right)^{k}\right\|\left\|\phi_{1}^{k}\right\|\right)<\left|x_{0}\right|^{2}\|F\| \frac{1}{2}\left(\sum_{k=0}^{\infty}\left\|\left(\phi_{1}^{T}\right)^{k}\right\|\right)\left(\sum_{k=0}^{\infty}\left\|\phi_{1}^{k}\right\|\right) \leq \\
& \leq \frac{1}{2}\left|x_{0}\right|^{2}\|F\| K_{\phi}^{2}<\infty
\end{aligned}
$$

Term 4: The formulation is simple and straightforward considering $\left|r_{k+2}\right|<E e^{-a(k+2)}$ and $\left|r_{k+2}\right| \leq E \quad \forall k$ :

$$
\begin{aligned}
& \frac{1}{2} \sum_{k=0}^{\infty}\left|r_{k+2}^{T} M_{\infty}^{T} F M_{\infty} r_{k+2}\right| \leq \frac{1}{2} \sum_{k=0}^{\infty}\left|r_{k+2}^{T}\right||| M_{\infty}^{T} F M_{\infty}||\left|r_{k+2}\right|< \\
& <\frac{1}{2} E\left\|M_{\infty}^{T} F M_{\infty}\right\| E \frac{1}{1-e^{-a}}<\infty
\end{aligned}
$$


This is the author version before final review of article published in European Journal of Control Vol. 39, January 2018, pp. 8-20

Terms 5, 6, 7, 11, 16: These terms can be considered together:

$$
\begin{aligned}
& \frac{1}{2} \sum_{k=0}^{\infty}\left|2 x_{0}^{T}\left(\phi_{1}^{T}\right)^{k}\left(F \Delta R_{k}+F L_{k}+F M_{\infty} r_{k+2}+Q H \Delta r_{k}+K_{x 2}^{T} R K_{r} \Delta \bar{r}_{k+2}\right)\right|< \\
& <\left|x_{0}^{T}\right|\left(\|F\| \phi_{U}\left\|B K_{r}\right\| 2 E \frac{1}{1-e^{-a}}+\|F\| \phi_{U}\left\|B K_{r_{\infty}}\right\| E \frac{1}{1-e^{-a}}+\right. \\
& \left.+\left\|F M_{\infty}\right\| E+\|Q H\| 2 E+\left\|K_{x 2}^{T} R K_{r}\right\| 2 E\right) \underbrace{\sum_{k=0}^{\infty}\left\|\left(\phi_{1}^{T}\right)^{k}\right\|}_{<K_{\phi}}<\infty
\end{aligned}
$$

Term 2: Considering that $\Delta R_{k}$ exists only for $k \geq 1$ and (B.3):

$$
\begin{aligned}
\frac{1}{2} \sum_{k=0}^{\infty}\left|\Delta R_{k}^{T} F \Delta R_{k}\right| \leq & \frac{1}{2} \sum_{k=1}^{\infty}\left|\Delta R_{k}^{T}\right|\|F\|\left|\Delta R_{k}\right| \leq \phi_{U}\left\|B K_{r}\right\| 2 E \frac{1}{1-e^{-a}}\|F\| \frac{1}{2} \sum_{k=1}^{\infty}\left|\Delta R_{k}\right| \\
\sum_{k=1}^{\infty}\left|\Delta R_{k}\right|= & \sum_{k=1}^{\infty}\left|\sum_{l=0}^{k-1}\left(\phi_{1}^{l} B K_{r} \Delta \bar{r}_{k+1}-l\right)\right|<\sum_{k=1}^{\infty} \sum_{l=0}^{k-1}\left\|\phi_{1}^{l}\right\|\left\|B K_{r}\right\| 2 E e^{-a(k-l)}= \\
= & \left\|B K_{r}\right\| 2 E e^{-a}+\left\|B K_{r}\right\|\left(2 E e^{-2 a}+\left\|\phi_{1}\right\| 2 E e^{-a}\right)+ \\
& +\left\|B K_{r}\right\|\left(2 E e^{-3 a}+\left\|\phi_{1}\right\| 2 E e^{-2 a}+\left\|\phi_{1}^{2}\right\| 2 E e^{-a}\right)+\ldots= \\
= & \lim _{N \rightarrow \infty} \sum_{j=0}^{N-1}\left(\sum_{l=0}^{j}\left\|\phi_{1}^{l}\right\|\right)\left\|B K_{r}\right\| 2 E e^{-(N-j) a}< \\
& <\lim _{N \rightarrow \infty}^{N-1} \sum_{j=0}^{\left(\sum_{l=0}^{\infty}\left\|\phi_{1}^{l}\right\|\right)}\left\|B K_{r}\right\| 2 E e^{-(N-j) a}< \\
& <K_{\phi}\left\|B K_{r}\right\| 2 E \sum_{j=0}^{\infty} e^{-j a}=K_{\phi}\left\|B K_{r}\right\| 2 E \frac{1}{1-e^{-a}}>\frac{1}{2} \sum_{k=1}^{\infty}\left|\Delta R_{k}\right| \\
\frac{1}{2} \sum_{k=0}^{\infty}\left|\Delta R_{k}^{T} F \Delta R_{k}\right|< & \phi_{U}\left\|B K_{r}\right\| 2 E \frac{1}{1-e^{-a}\|F\|} \frac{1}{2} K_{\phi}\left\|B K_{r}\right\| 2 E \frac{1}{1-e^{-a}}<\infty
\end{aligned}
$$

Term 3: Formally the same as Term 2, except for one needs to consider (B.4):

$$
\frac{1}{2} \sum_{k=0}^{\infty}\left|L_{k}^{T} F L_{k}\right|<\phi_{U}\left\|B K_{r_{\infty}}\right\| E \frac{1}{1-e^{-a}}\|F\| \frac{1}{2} K_{\phi}\left\|B K_{r_{\infty}}\right\| E \frac{1}{1-e^{-a}}<\infty
$$


This is the author version before final review of article published in European Journal of Control Vol. 39, January 2018, pp. 8-20

Term 8: Formally the same as Term 2:

$$
\frac{1}{2} \sum_{k=0}^{\infty} 2\left|\Delta R_{k}^{T} F L_{k}\right|<\phi_{U}\left\|B K_{r}\right\| 2 E \frac{1}{1-e^{-a}}\|F\| K_{\phi}\left\|B K_{r_{\infty}}\right\| E \frac{1}{1-e^{-a}}<\infty
$$

Terms 9, 12, 17: These terms can be considered together:

$$
\begin{aligned}
& \frac{1}{2} \sum_{k=0}^{\infty}\left|2 \Delta R_{k}^{T}\left(F M_{\infty} r_{k+2}+Q H \Delta r_{k}+K_{x 2}^{T} R K_{r} \Delta \bar{r}_{k+2}\right)\right|< \\
& <\phi_{U}\left\|B K_{r}\right\| 2 E \frac{1}{1-e^{-a}}\left(\left\|F M_{\infty}\right\| E+\|Q H\| 2 E+\left\|K_{x 2}^{T} R K_{r}\right\| 2 E\right) \frac{1}{1-e^{-a}}<\infty
\end{aligned}
$$

Terms 10, 13, 18: These terms can be considered together:

$$
\begin{aligned}
& \frac{1}{2} \sum_{k=0}^{\infty}\left|2 L_{k}^{T}\left(F M_{\infty} r_{k+2}+Q H \Delta r_{k}+K_{x 2}^{T} R K_{r} \Delta \bar{r}_{k+2}\right)\right|< \\
& <\phi_{U}\left\|B K_{r_{\infty}}\right\| E \frac{1}{1-e^{-a}}\left(\left\|F M_{\infty}\right\| E+\|Q H\| 2 E+\left\|K_{x 2}^{T} R K_{r}\right\| 2 E\right) \frac{1}{1-e^{-a}}<\infty
\end{aligned}
$$

Terms 14, 19: These terms can be considered together:

$$
\begin{aligned}
& \frac{1}{2} \sum_{k=0}^{\infty}\left|2 r_{k+2}^{T} M_{\infty}^{T}\left(Q H \Delta r_{k}+K_{x 2}^{T} R K_{r} \Delta \bar{r}_{k+2}\right)\right|< \\
& <E\left(\left\|M_{\infty}^{T} Q H\right\| 2 E+\left\|M_{\infty}^{T} K_{x 2}^{T} R K_{r}\right\| 2 E\right) \frac{1}{1-e^{-a}}<\infty
\end{aligned}
$$

Term 15:

$$
\frac{1}{2} \sum_{k=0}^{\infty}\left|\Delta r_{k}^{T} H^{T} Q H \Delta r_{k}\right|<2 E\left\|H^{T} Q H\right\| 2 E \frac{1}{1-e^{-a}}<\infty
$$

Term 20: Formally it is the same as Term 15:

$$
\frac{1}{2} \sum_{k=0}^{\infty}\left|\Delta \bar{r}_{k+2}^{T} K_{r}^{T} R K_{r} \Delta \bar{r}_{k+2}\right|<2 E e^{-a}\left\|K_{r}^{T} R K_{r}\right\| 2 E \frac{1}{1-e^{-a}}<\infty
$$


This is the author version before final review of article published in European Journal of Control Vol. 39, January 2018, pp. 8-20

\section{Appendix C. Tuning weights in the different cases}

This section summarizes the obtained best tuning weights for the presented different methods. The list of notations applied in the tables in the following sections is:

- LQT: infinite horizon linear quadratic tracking controller

- SPT: nonzero set point tracker

- PV: preview tracker

- MPC: model predictive control

- NEX: non-extrapolating solutions with two-step (i. e. $r_{k+1}$ and $r_{k+2}$ ) preview

- EX: extrapolating solutions with only one-step $\left(r_{k+1}\right)$ preview

- minC: cases tuned for minimum cost function value $\left(J_{S}\right)$

- minT: cases tuned for minimum average tracking error

Table C.1: Best $Q_{2}$ weights in the different cases

\begin{tabular}{ccccc}
\hline Ref. type / method / goal & LQT & SPT & PV & MPC \\
\hline \hline doublet / NEX / minC & 10 & 10 & 10 & 10 \\
\hline doublet/ NEX / minT & 10 & 10 & 10 & 320 \\
\hline \hline doublet / EX / minC & 10 & 10 & 10 & 10 \\
\hline doublet/ EX / minT & 10 & 10 & 10 & 320 \\
\hline \hline chirp / NEX / minC & 1530 & 1150 & 1530 & 210 \\
\hline chirp / NEX / minT & 238370 & $10^{7}$ & 238370 & 54380 \\
\hline \hline chirp / EX / minC & 10 & 15480 & 4320 & 210 \\
\hline chirp / EX / minT & 10000 & $10^{8}$ & 422910 & 57450 \\
\hline \hline
\end{tabular}


This is the author version before final review of article published in European Journal of Control Vol. 39, January 2018, pp. 8-20

\section{Appendix D. Numerical results with the different tracking solutions}

This section summarizes the obtained performance metrics with the different tracking solutions. The applied abbreviations are defined in Section Appendix C.

Table D.1: Results of doublet tracking with NEX methods

\begin{tabular}{ccccc}
\hline Controller / goal & $\theta_{e}\left[{ }^{\circ}\right]$ & $J_{S}$ & $J_{S 1}$ & $J_{s 2}$ \\
\hline \hline LQT & 1.31207 & 79.989 & 677.806 & 51545.039 \\
\hline SPT & 1.31207 & 79.989 & 677.806 & 51545.04 \\
\hline PV & 1.31207 & 79.989 & 677.806 & 51545.039 \\
\hline MPC / minC & 1.6239 & 83.1 & 673.183 & 54892.74 \\
\hline MPC / minT & 1.4309 & 2538.524 & 700.291 & 52837.053 \\
\hline
\end{tabular}

Table D.2: Results of doublet tracking with EX methods

\begin{tabular}{ccccc}
\hline Controller / goal & $\theta_{e}\left[^{\circ}\right]$ & $J_{s}$ & $J_{s 1}$ & $J_{s 2}$ \\
\hline \hline LQT & 1.35206 & 83.324 & 677.571 & 51544.697 \\
\hline SPT & 1.35206 & 83.324 & 677.571 & 51544.719 \\
\hline PV & 1.35206 & 83.324 & 677.571 & 51544.719 \\
\hline MPC / minC & 1.66386 & 86.45 & 672.95 & 54892.097 \\
\hline MPC / minT & 1.47095 & 2645.23 & 700.056 & 52836.557 \\
\hline
\end{tabular}


This is the author version before final review of article published in European Journal of Control Vol. 39, January 2018, pp. 8-20

Table D.3: Results of chirp tracking with NEX methods

\begin{tabular}{cccccc}
\hline Controller / goal & $\theta_{e}\left[^{\circ}\right]$ & $J_{s}$ & $J_{s 1}$ & $J_{s 2}$ & $\tau[s]$ \\
\hline \hline $\mathrm{LQT} / \mathrm{minC}$ & 0.19322 & 8.361 & 2376.55 & 193798.8 & 0.01 \\
\hline $\mathrm{LQT} / \mathrm{minT}$ & 0.098848 & 9064.3 & 2377.343 & 193814.919 & 0.01 \\
\hline \hline $\mathrm{SPT} / \mathrm{minC}$ & 0.19291 & 7.627 & 2376.53 & 193806.58 & 0.01 \\
\hline $\mathrm{SPT} / \mathrm{minT}$ & 0.005124 & 1178583.28 & 2378.719 & 193853.377 & 0.0 \\
\hline \hline $\mathrm{PV} / \mathrm{minC}$ & 0.19322 & 8.361 & 2376.55 & 193798.8 & 0.01 \\
\hline $\mathrm{PV} / \mathrm{minT}$ & 0.09885 & 9064.299 & 2377.343 & 193814.919 & 0.01 \\
\hline \hline $\mathrm{MPC} / \mathrm{minC}$ & 0.19567 & 6.7394 & 2377.676 & 195574.1 & 0.01 \\
\hline $\mathrm{MPC} / \mathrm{minT}$ & 0.11039 & 1702.624 & 2377.009 & 193815.322 & 0.01 \\
\hline
\end{tabular}

Table D.4: Results of chirp tracking with EX methods

\begin{tabular}{cccccc}
\hline Controller / goal & $\theta_{e}\left[^{\circ}\right]$ & $J_{s}$ & $J_{s 1}$ & $J_{s 2}$ & $\tau[s]$ \\
\hline \hline $\mathrm{LQT} / \mathrm{minC}$ & 0.14127 & 5.6076 & 2380.506 & 196863.676 & 0.01 \\
\hline $\mathrm{LQT} / \mathrm{minT}$ & 0.11111 & 313.898 & 2377.083 & 193927.027 & 0.01 \\
\hline \hline $\mathrm{SPT} / \mathrm{minC}$ & 0.19331 & 37.087 & 2376.56 & 193805.9 & 0.01 \\
\hline $\mathrm{SPT} / \mathrm{minT}$ & 0.1125 & 3004992 & 2377.036 & 193830.409 & 0.01 \\
\hline \hline $\mathrm{PV} / \mathrm{minC}$ & 0.19331 & 14.122 & 2376.583 & 193839.92 & 0.01 \\
\hline $\mathrm{PV} / \mathrm{minT}$ & 0.08422 & 20134.259 & 2378.112 & 193865.886 & 0.01 \\
\hline \hline $\mathrm{MPC} / \mathrm{minC}$ & 0.19706 & 6.826 & 2377.804 & 195664.033 & 0.01 \\
\hline $\mathrm{MPC} / \mathrm{minT}$ & 0.11042 & 1798.826 & 2377.048 & 193873.492 & 0.01 \\
\hline
\end{tabular}

